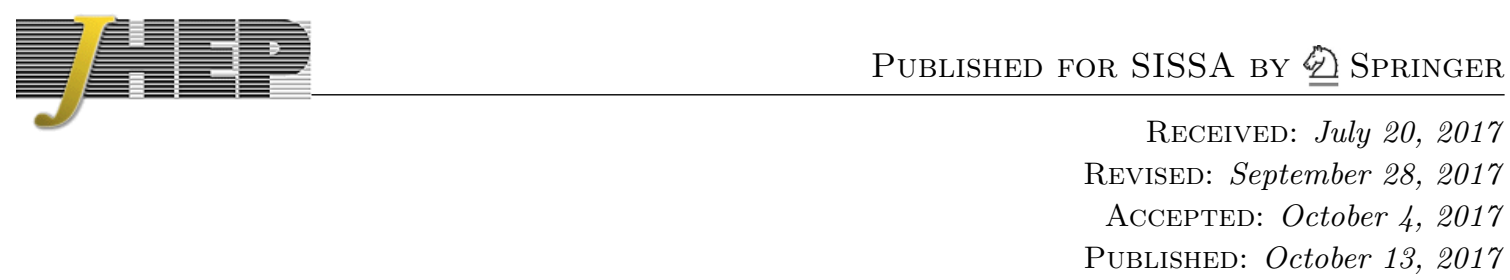

\title{
The leptoquark hunter's guide: pair production
}

\author{
Bastian Diaz, ${ }^{a}$ Martin Schmaltz ${ }^{b}$ and Yi-Ming Zhong ${ }^{b}$ \\ ${ }^{a}$ Departamento de Fisica and Centro Cientfico-Tecnologico de Valparaiso, \\ Universidad Tecnica Federico Santa Maria, Casilla 110-V, Valparaiso, Chile \\ ${ }^{b}$ Physics Department, Boston University, \\ Boston, MA 02215, U.S.A. \\ E-mail: bastian.diaz@alumnos.usm.cl, schmaltz@bu.edu, ymzhong@bu.edu
}

ABSTRACT: Leptoquarks occur in many new physics scenarios and could be the next big discovery at the LHC. The purpose of this paper is to point out that a model-independent search strategy covering all possible leptoquarks is possible and has not yet been fully exploited. To be systematic we organize the possible leptoquark final states according to a leptoquark matrix with entries corresponding to nine experimentally distinguishable leptoquark decays: any of \{light-jet, $b$-jet, top $\}$ with any of $\{$ neutrino, $e / \mu, \tau\}$. The 9 possibilities can be explored in a largely model-independent fashion with pair-production of leptoquarks at the LHC. We review the status of experimental searches for the 9 components of the leptoquark matrix, pointing out which 3 have not been adequately covered. We plead that experimenters publish bounds on leptoquark cross sections as functions of mass for as wide a range of leptoquark masses as possible. Such bounds are essential for reliable recasts to general leptoquark models. To demonstrate the utility of the leptoquark matrix approach we collect and summarize searches with the same final states as leptoquark pair production and use them to derive bounds on a complete set of Minimal Leptoquark models which span all possible flavor and gauge representations for scalar and vector leptoquarks.

Keywords: Beyond Standard Model, Hadron-Hadron scattering (experiments)

ARXIV EPRINT: 1706.05033 


\section{Contents}

1 Introduction and overview 1

2 Minimal leptoquark models $\quad 9$

3 Leptoquark searches organized by the LQ matrix 13

$3.1 \nu j, \nu b$ and $\nu t \quad 13$

$\begin{array}{ll}3.2 \mathrm{ej} \text { and } \mu j & 16\end{array}$

$3.3 e b$ and $\mu b \quad 17$

$\begin{array}{ll}3.4 \text { et and } \mu t & 18\end{array}$

$\begin{array}{lll}3.5 & \tau j & 21\end{array}$

$\begin{array}{lll}3.6 & \tau b & 21\end{array}$

$\begin{array}{lll}3.7 & \tau t & 21\end{array}$

4 Discussion and summary $\quad 23$

$\begin{array}{ll}\text { A Notation } & 25\end{array}$

B Comparison between vector and scalar LQ searches 25

$\begin{array}{ll}\text { C A vector leptoquark model } & 26\end{array}$

\section{Introduction and overview}

A leptoquark (LQ) $[1,2]$ is a particle with a coupling which allows it to decay to a quark (or anti-quark) and a lepton, figure 1. It carries color and electric charge, and possibly also weak charges. Because of their color, leptoquarks can be pair-produced as LQ antiLQ pairs with large QCD cross sections. Searches based on this production mode with subsequent decay, $p p \rightarrow \phi \bar{\phi} \rightarrow(l q)(\bar{l} \bar{q})$, are the subject of this paper (we plan to address single LQ production in a future publication see [3], searches for single LQ production have a long history at HERA, for reviews see e.g. $[2,4])$. Here $\phi$ and $\bar{\phi}$ are the leptoquark and its antiparticle, $q$ can be any Standard Model (SM) quark or antiquark, $l$ is any lepton, and $\bar{q}$ and $\bar{l}$ are the corresponding antiparticles. We use parenthesis to indicate which final state particles reconstruct resonances. Our goal is to provide a simple organizing principle which makes it straightforward to systematically search for all possible leptoquarks. The idea is that we identify a minimum set of independent final states which must be searched for. These final states can be arranged into a $3 \times 3$ matrix which we call the "leptoquark final state matrix", or simply "LQ matrix", figure 1. For each final state, what is needed from experiment is an upper bound on the cross section times branching fraction as a function of LQ mass. Lower bounds on the mass of an arbitrary leptoquark can then be obtained 

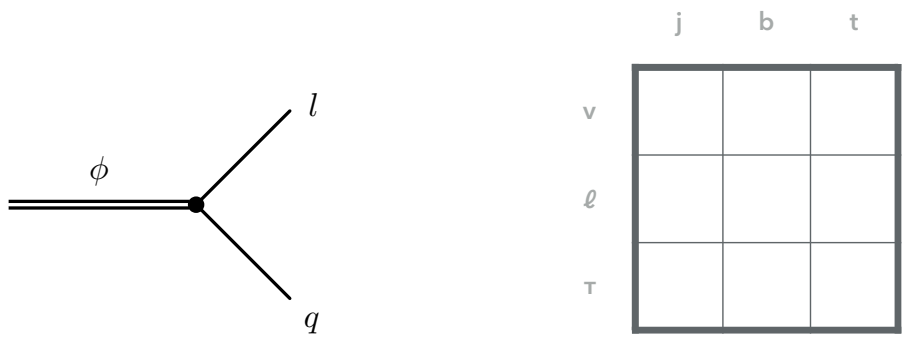

Figure 1. (Left) LQ coupling to quark $q$ and lepton $l$ (see appendix A for notations used in the paper). (Right) LQ matrix. Each entry of the matrix represents one of the experimentally distinguishable leptoquark decays. Rows label leptonic decay products, columns label hadronic decay products. A more precise definition of the LQ matrix is given in the text. For a summary of existing LHC searches corresponding to each matrix element see section 3.

by calculating the theoretical cross section times branching fraction into the final states in the LQ matrix and comparing to the experimental cross section bounds.

This paper consists of two complementary parts. In Part 1, we introduce the LQ matrix and show that bounds on the cross sections into each of the 9 final states of the matrix are both efficient and sufficient for searching for all possible LQs. In Part 2 we demonstrate the utility of this approach. We collect the best currently available cross section bounds from LHC searches organized by which element of the LQ matrix they cover and use them to put bounds on a complete set of "Minimal Leptoquark" (MLQ) models. The MLQs include scalar and vector LQs with all possible flavor, charge, and isospin quantum numbers.

We begin with a few words on the theoretical motivation for leptoquarks. LQs are predicted in many physics beyond the SM scenarios. Vector LQs may be related to unification, squarks automatically become scalar LQs in supersymmetry (SUSY) with $R$-parity violation (RPV), and LQs arise in models in which quarks and leptons are composites of the same underlying dynamics. Recently, additional motivation for LQs arrived from a number of anomalies observed in $B$ meson decays [5, 6] which could be explained in models with leptoquarks of $\mathrm{TeV}$ scale masses. Leptoquarks with couplings to muons might also be relevant to the persistent anomaly in the muon anomalous magnetic moment [7]. On the other hand, there are no convincing theoretical arguments for preferring a particular pattern of leptoquark flavors or quantum numbers, and the experimental hints, however intriguing, may still change or disappear. This motivates a systematic approach to search for a minimal but sufficient set of final states which can discover LQs with arbitrary decays to quarks and leptons.

What are the possible LQ final states? By definition, a leptoquark can decay to any of the six SM quarks with any of the three charged leptons or three neutrinos. The quarks and leptons can be particle or antiparticle and left- or right-handed (we allow for right-handed neutrinos), corresponding to hundreds of different possibilities. However, many of these are covered by the same searches. For example, left- and right-handed final state particles are generally not distinguishable. Light quarks and antiquarks $u, d, s, c, \bar{u}, \bar{d}, \bar{s}, \bar{c}$ from 
leptoquark decays all show up as light-jets. ${ }^{1}$ Heavy quarks, $t$ and $b$ can be distinguished. For leptons, we have three distinct charged leptons but neutrino flavor is not observable in leptoquark decays. Thus we arrive at a matrix of nine distinct final states from the decay of a single LQ, three "flavors" of quarks $j, b, t$ times three "flavors" of leptons ${ }^{2} \nu, \ell(=e / \mu)$, $\tau$, see figure $1 b$.

To obtain the final states of pair production we must consider the decay of the LQ and its antiparticle. For a LQ with a unique coupling to a quark-lepton bilinear $q l$, the decay of the LQ anti-LQ pair is also unique to the "symmetric" final state $(l q)(\bar{l} \bar{q})$. Enumerating all possible such symmetric final states is trivial, they are determined by adding the corresponding antiparticles to the final states of a single LQ. Thus we can use the same $3 \times 3$ LQ matrix to classify the distinct symmetric final states.

However, when LQs have multiple decay channels then LQ pair production also produces "asymmetric" final states which are not covered by the classification in terms of a single $3 \times 3$ matrix. We now argue that the symmetric final states are all we need. Consider for simplicity the case where a LQ couples to two quark-lepton pairs, $l q$ and $l^{\prime} q^{\prime}$. We obtain the pair production final states:

$$
\begin{array}{cc}
\text { symmetric: } & (l q)(\bar{l} \bar{q})+\left(l^{\prime} q^{\prime}\right)\left(\bar{l}^{\prime} \bar{q}^{\prime}\right) \\
\text { asymmetric : } & (l q)\left(\bar{l}^{\prime} \bar{q}^{\prime}\right)+\left(l^{\prime} q^{\prime}\right)(\bar{l} \bar{q})
\end{array}
$$

The two symmetric ones are contained in the LQ matrix classification, the asymmetric ones are not. But here is the point: all leptoquarks which produce asymmetric final states necessarily also produce symmetric final states. Therefore the symmetric final states are sufficient to search for all possible leptoquarks. Moreover, one might expect the couplings of leptoquarks to different quark-lepton pairs to not all be the same. Then the branching fraction to the quark-lepton pair with the largest coupling $l q$ will dominate the decays, and the branching fraction to the symmetric state $(l q)(\bar{l} \bar{q})$ will be largest. To sum up, symmetric final states from pair production of LQs can be represented by the LQ matrix. They are sufficient for searching for all possible leptoquarks. When LQ couplings to quarklepton pairs are at least somewhat hierarchical, a symmetric final state will usually also yield the most sensitive search.

An interesting special case arises when a symmetry relates couplings of the leptoquark to different quark-lepton pairs. In fact, isospin, $\mathrm{SU}(2)_{\text {weak }}$, is such a symmetry. Consider for concreteness an $\mathrm{SU}(2)_{\text {weak }}$ singlet scalar leptoquark which couples to the $\mathrm{SU}(2)_{\text {weak }}$ doublets $q_{L}$ and $l_{L}$, both of the first generation. $\mathrm{SU}(2)_{\text {weak }}$ enforces that the coupling of the LQ is to the combination $d \nu-u e^{-}$so that pair production gives rise to the four possible final states $(\nu d)(\bar{\nu} \bar{d})+\left(e^{-} u\right)\left(e^{+} \bar{u}\right)+(\nu d)\left(e^{+} \bar{u}\right)+\left(e^{-} u\right)(\nu \bar{d})$, each with $25 \%$ branching fraction. One could search for this leptoquark with the symmetric LQ matrix final states $\left(e^{-} j\right)\left(e^{+} j\right)$ and $j j \mathbb{E}_{\mathrm{T}}$ or with non-matrix final states $\left(e^{+} j\right)\left(j \mathbb{E}_{\mathrm{T}}\right)$ and $\left(e^{-} j\right)\left(j \mathbb{E}_{\mathrm{T}}\right)$. However

\footnotetext{
${ }^{1} c$-tagging is not yet efficient enough to improve leptoquark searches. We show this with an example in section 3.1.

${ }^{2}$ Note that it is customary in LHC searches to refer to electrons or muons as light leptons or " $\ell$ " because search strategies for them are similar; we reflect this custom by assigning $e / \mu$ to a single row, $\ell$. Of course, one could instead work with a $4 \times 3$ matrix.
} 
even in this case where no single symmetric final state dominates the "easiest" and likely most sensitive search is the symmetric search for $\left(e^{-} j\right)\left(e^{+} j\right)$. We will discuss many more examples with multiplicities of final states from $\mathrm{SU}(2)_{\text {weak }}$ in section 3 .

So far, we have argued that in order to search for all possible LQs, it is sufficient for experiments to cover 9 symmetric final states which are in one-to-one correspondence with elements of the LQ matrix. What information do we need from each of these 9 searches in order to be able to put bounds on arbitrary leptoquarks?

In order to maximize sensitivity, LHC leptoquark searches adjust their cuts as a function of the mass of LQs that are being searched for. In addition, signal efficiencies depend on the LQ mass. Therefore, the natural result of a leptoquark search is a "Brazil plot" which shows the experimentally observed upper bound on the allowed signal cross section times branching fraction as a function of LQ mass (along with the range of expected bounds). This plot of the upper bound on $\sigma \times B r$ for each final state in the LQ matrix is precisely the result which is needed from experiment. With the 9 plots from each of the LQ matrix searches anyone can then easily determine a lower bound on the mass of their favorite leptoquark by comparing their theoretical $\sigma \times B r$ as a function of mass to the bound. This is true for LQs of arbitrary spin, charge, or couplings.

This last claim requires further examination. In order to determine the bound on $\sigma \times B r$ experimenters need to divide the observed bound on the signal cross section for events in a signal region by the efficiency of signal events to pass the signal region cuts. In general, this signal efficiency can depend on many properties of the signal under consideration, and one may worry that model-independent bounds on $\sigma \times B r$ cannot be obtained. Fortunately, the situation for symmetric LQ searches is nice. To fairly good precision (better than 10 $\%$ ) the signal efficiency only depends on the mass of the leptoquark. The basic reason for this model independence is easy to understand: current LQ bounds already require masses well above $500 \mathrm{GeV}$ and center of mass energies in excess of $1 \mathrm{TeV}$. Thus leptoquarks are produced with only moderate boosts. Furthermore they two-body-decay directly to SM particles, yielding highly energetic widely separated SM particles with approximately isotropic distributions. Thus signal efficiencies for standard kinematic cuts are high and only very weakly dependent on any details of the LQ model other than the LQ mass. Two concerns which could have invalidated this argument are dependence on LQ spin and width, we discuss them in turn.

Spin: leptoquarks can be spin 0 or 1, i.e., scalars or vectors. In either case the cross section is dominated by gluon-gluon interactions. Nevertheless, scalars from quark-antiquark initial states are produced in a $p$-wave, implying that this cross section is more suppressed near threshold, so that typical $q \bar{q}$ initiated scalar LQ events have more energy. Thus we might expect the efficiencies of $p_{T}$ cuts to be slightly higher for scalars. This is a small effect because the bulk of the production is from gluon-gluon interactions and even LQs decaying at rest would give their decay products sufficient momentum to pass basic $p_{T}$ cuts. More important are angular distributions resulting from LQ spin. Because of the large LQ mass, decay products are well-isolated and approximately back-to-back. However the pseudo-rapidity, $\eta$, distribution of vector LQs is more forward than for the scalars, causing 


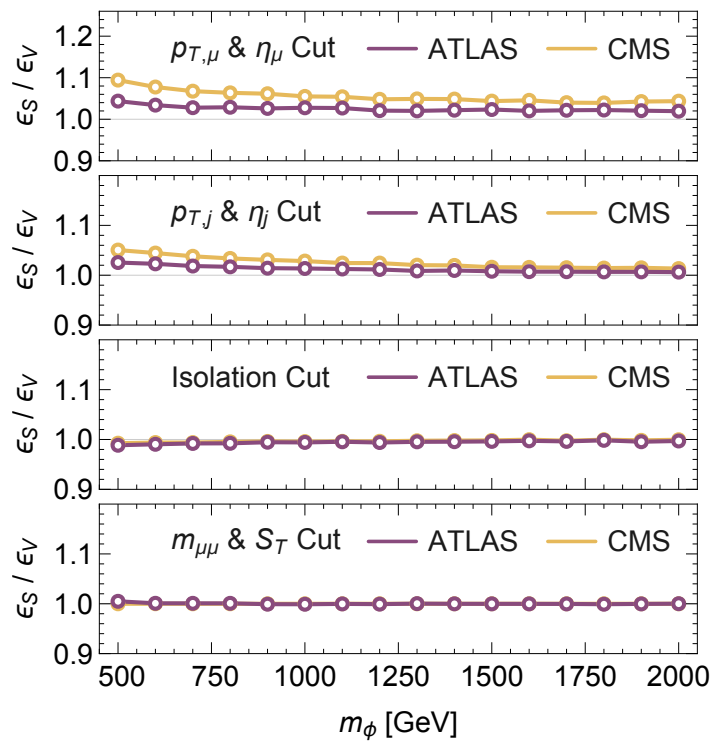

Figure 2. Ratios of signal efficiencies $\epsilon_{S} / \epsilon_{V}$ for pair-produced scalar and vector LQs with various masses. The LQ decays are to muons and jets. The panels show the relative efficiencies of a series of selection cuts from recent ATLAS [8] and CMS [9] analyses. Details of the cuts and plots of phase space distributions are in appendix B.

the efficiency of especially lepton $\eta$ cuts to be lower in the vector case. But this effect is also small. To demonstrate this we show the ratio of the two signal efficiencies, $\epsilon_{S} / \epsilon_{V}$, as a function of mass for the analysis cuts used in two typical ATLAS [8] and CMS [9] analyses in figure 2 (details are shown in appendix B). This analysis is for LQs decaying to muons and light quarks and with signal simulated at the parton level. One sees that for LQ masses in the range of current bounds $(800 \mathrm{GeV}-2 \mathrm{TeV})$ the difference between scalar and vector efficiencies is always smaller than $10 \%$.

We conclude that searches with cuts optimized for scalar LQ detection are also very sensitive to vectors and the differences in efficiencies are small enough that we can ignore them. An analysis aiming for higher precision could account for the small difference in efficiencies between vectors and scalars by determining separate cross section bounds for the two cases.

Width: for leptoquarks with very large couplings $(\gg 1)$ to quarks and leptons the width becomes large and a significant number of events may have one or both LQs off-shell. In that case signal efficiencies can become much more model-dependent. However large couplings to quarks and leptons of the first and second generation for $\mathrm{TeV}$ scale leptoquarks are ruled out from single leptoquark production, $e^{+} e^{-}$collisions, and precision electroweak constraints. Large couplings to third generation particles remain a possibility which could introduce some model dependence. We ignore this special possibility and assume that LQ widths are smaller than $10 \%$ so that on-shell LQ production dominates.

To summarize our results so far, we have argued that in order to search for arbitrary LQs it is sufficient to consider 9 final states corresponding to the LQ matrix. Experiments 

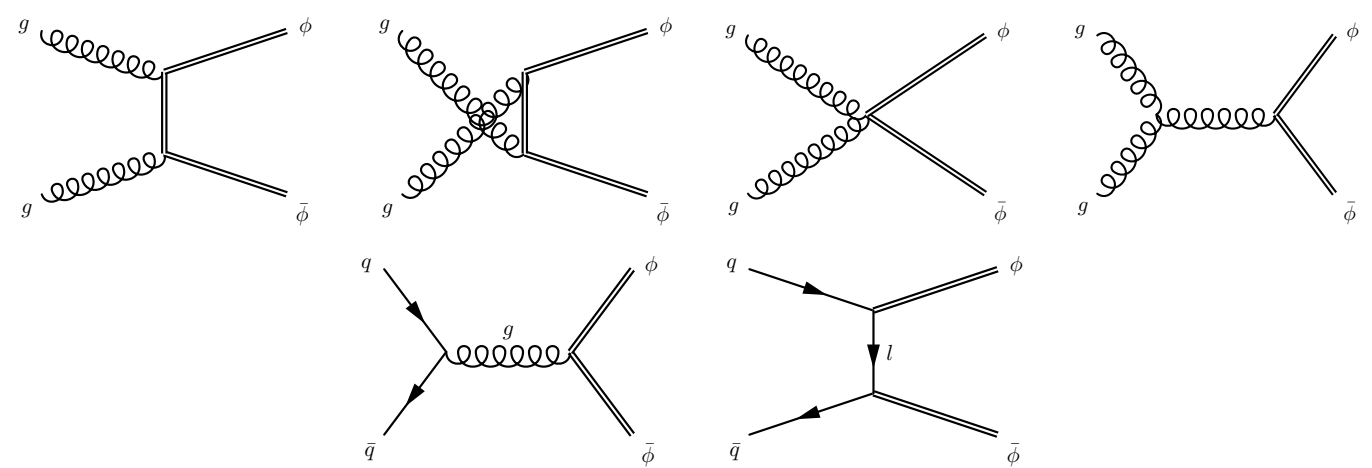

Figure 3. Diagrams for leptoquark pair production: gluon-initiated (upper row), quark-initiated (lower-left), and diagram proportional to the square of the leptoquark-lepton-quark coupling, $\lambda^{2}$ (lower-right).

should provide the upper bound on the cross section times branching fraction as a function of LQ mass for each of the different final states (Brazil plot). The signal efficiencies needed to produce the cross section bounds are largely model independent and can be determined from Monte Carlo (MC) for any convenient leptoquark implementation (scalar or vector with arbitrary couplings). Armed with cross section bounds for each LQ matrix final state one can determine lower mass bounds on arbitrary LQs by computing the cross section times branching fractions and comparing to the bounds.

The leading Feynman diagrams contributing to the LQ pair-production cross section are shown in figure 3 .

Gluon-initiated diagrams dominate for all but the largest LQ masses. The diagram proportional to the square of the leptoquark-lepton-quark coupling $\lambda$ is model dependent but it is never important for pair production. This is because once the coupling is large enough to make a difference for pair production it also contributes to single LQ production at the same order in $\lambda$ and single production will yield a stronger limit [3, 10, 11]. Therefore we will ignore this diagram in the following.

LQ cross sections times branching fractions into the dominant final states are large because LQs carry color and are strongly produced. Choosing for example a leptoquark mass of $1 \mathrm{TeV}$, hundreds of scalar leptoquarks or thousands of vector leptoquarks (would) have been produced with the $\sim 36 \mathrm{fb}^{-1}$ data of $13 \mathrm{TeV}$ accumulated in 2016 at the LHC, see figure $4 .^{3}$ The scalar LQ cross section is model independent when the last diagram in figure 3 is negligible. The vector case has a subtlety related to unitarity of theories with massive vectors. A consistent theory must have additional states beyond the LQ with masses not too far above the LQ mass. For computing the vector cross section in the figure we used a minimal model implementation described in appendix $\mathrm{C}$. The model has a massive gluon partner, $g^{\prime}$, which contributes to LQ pair production. Varying the mass of the gluon partner across its theoretically allowed range we obtain the two limiting cross sections labeled $m_{g^{\prime}} / m_{V}=0, \sqrt{2}$ in figure 4 .

\footnotetext{
${ }^{3}$ Leptoquarks carry color, charge, baryon and lepton numbers. Hence they cannot be hidden with invisible or fully hadronic decays.
} 


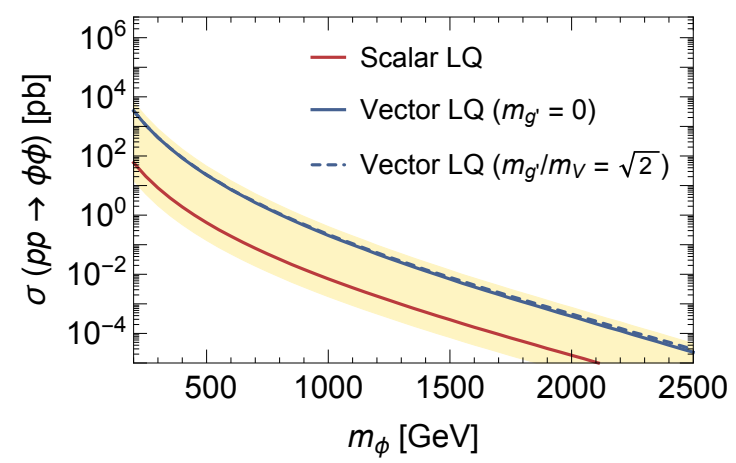

Figure 4. Pair-production cross sections of LQs. Predictions are leading order (LO) with $K$-factors taken from $[12,13]$ ( $K$-factors have also been discussed in [14-16]). LO cross section were produced with CTEQ6 parton distribution functions and the renormalization scale $\mu=m_{\phi}$. For simplicity, we applied the $K$-factors for scalars $S$ also in the vector $V$ case. For details of the vector model see appendix C. The cream band is explained in section 2 .

We now show as an example how to obtain lower limits on LQ masses using the LQ matrix final state $\left(\mu^{+} j\right)\left(\mu^{-} j\right)$, which is obtained from pair-production and decay of a LQ decaying to muons and jets. A nice analysis for this final state has been presented by CMS [9]. CMS provided a 95\% confidence level (CL) upper bound on the cross section times branching fraction to this final state which we represent with the solid black curve in the left panel of figure 5. Also shown as colorful lines are the theoretical cross sections times branching fractions for a number of Minimal LQ models which we define in the next section. Four of the models have scalar LQs with pair production cross sections times branching fraction ranging from $0.25 \sigma_{S}$ to $2 \sigma_{S}$. Here $\sigma_{S}$ is the "fiducial" scalar cross section shown in figure 4 . The factors $0.25,1,1.25,2$ correspond to multiplicity factors and branching fractions which will also be explained in section 2. We just use them as examples to make the point that there are many different LQ models which can contribute to this final state. We can easily obtain lower bounds on the different MLQs' masses by using the plot of the experimental cross section bound. For example, the red leptoquark labeled $\sigma_{S}$ is excluded up to $1.17 \mathrm{TeV}$. This leptoquark was the designated target of the CMS search and the limit $1.17 \mathrm{TeV}$ can also be found in [9]. However none of the other MLQs were considered explicitly by CMS, and had the collaboration decided to only publish the mass bound a simple determination of mass bounds for all LQs would have been impossible.

Unfortunately, while CMS did provide the very useful plot of the 95\% CL cross section limit they did not extend the mass reach of their plot far enough in the leptoquark mass to allow a reliable extrapolation to vector leptoquarks which have much larger cross sections. In absence of a cross section limit provided by experimenters we are forced to guess. In this case it seems reasonable to guess that the cross section limit flattens out for large LQ masses because signal efficiencies from very heavy LQs approach saturation. Having made this guess (the dotted black line extension of the experimental limit) we can then put lower bounds on the masses of the four vector MLQ models. However, we emphasize that these mass limits are estimates based on our guess for how the sensitivity of the CMS search might continue to higher masses. 

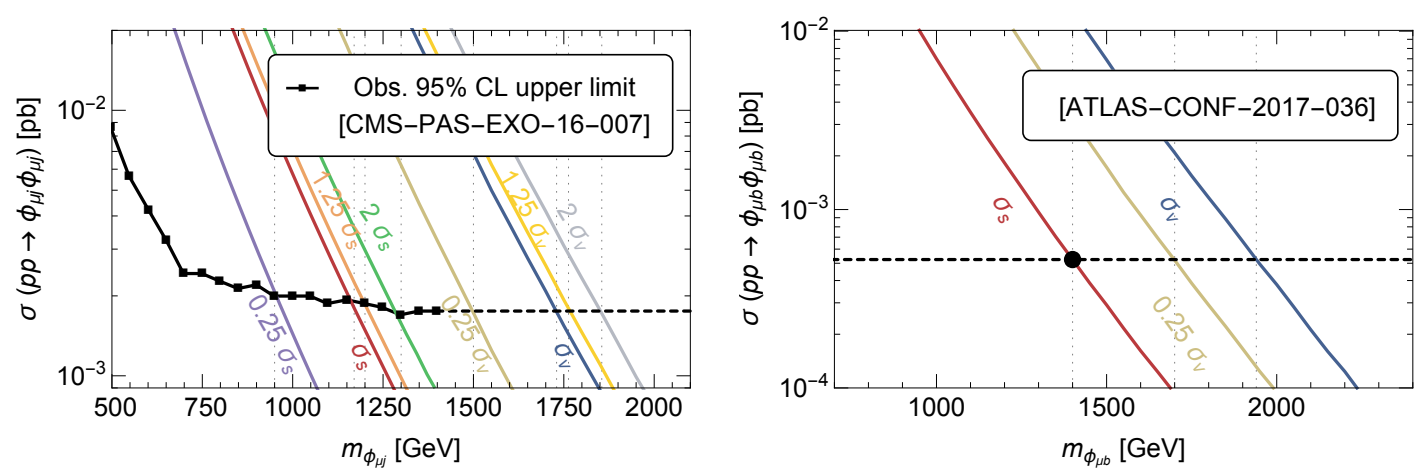

Figure 5. (Left plot) Bounds on scalar and vector leptoquarks which decay to $(\mu j)(\mu j)$ final states. The observed 95\% CL upper limit on the production cross section times branching fraction (black solid line) is taken from a search for leptoquarks in this final state at CMS [9]. The search stopped at $1.4 \mathrm{TeV}$. In order to obtain a plausible estimate for the mass limit on vector LQs we extended the cross section limit beyond $1.4 \mathrm{TeV}$ by assuming that it becomes mass-independent (black dashed line). The theoretical cross sections for different MLQs which can decay to $(\mu j)(\mu j)$ final states are shown as colored lines and labeled with corresponding cross sections. The intersects (vertical dotted lines) determine the lower limits on the MLQ masses. (Right plot) Bounds on scalar and vector MLQs which decay into $(\mu b)(\mu b)$ final states. Since only a 95\% CL on the stop/leptoquark mass (black dot) is provided in [17] we cannot derive rigorous mass bounds on other LQs. To obtain an estimate, we assume that the experimental upper limit on the cross section is flat across the LQ mass range and agrees with the value for the scalar fiducial cross section, $\sigma_{S}$, at the stop/leptoquark mass limit (black dashed horizontal line). Cross sections for other MLQs that can decay into $(\mu b)(\mu b)$ final states are shown as colored lines and labeled with their cross sections. The intersects (vertical dotted lines) determine our estimates for lower limits on the MLQ masses.

The situation depicted in the right panel of figure 5 is less satisfactory. This plot corresponds to the LQ matrix search for $\left(\mu^{-} b\right)\left(\mu^{+} \bar{b}\right)$. ATLAS performed this search as a SUSY search for RPV stop decays and obtained an impressive lower mass bound of $1.4 \mathrm{TeV}$. This RPV squark is identical in production and decay to a particular scalar leptoquark, and therefore this mass bound can be directly applied to the scalar leptoquark model. Given this bound and our knowledge of the theoretical cross section times branching fraction for this leptoquark we can infer that ATLAS found a 95\% CL upper bound of $4.5 \times 10^{-4}$ $\mathrm{pb}$ on the signal cross section for a LQ with mass $1.4 \mathrm{TeV}$ (indicated with the black dot in figure 5). Unfortunately, that is all the information we have. From this one data point it is not possible to infer bounds on the masses of any other LQ models with differing cross sections or branching fractions. The best we can do is make the assumption that perhaps the $95 \%$ CL limit on the cross section is approximately mass independent (as it appears to be for the CMS search in the left panel for larger LQ masses). Using our guess for the cross section bound (horizontal dashed line) we can now provide estimates for the mass bounds of other MLQ models by reading off the intersects of the cross section predictions with the extrapolated cross section bound.

In the remainder of the paper we present results for LHC searches for all 9 of the LQ matrix elements. We found fully satisfactory cross section limit plots in six cases, 


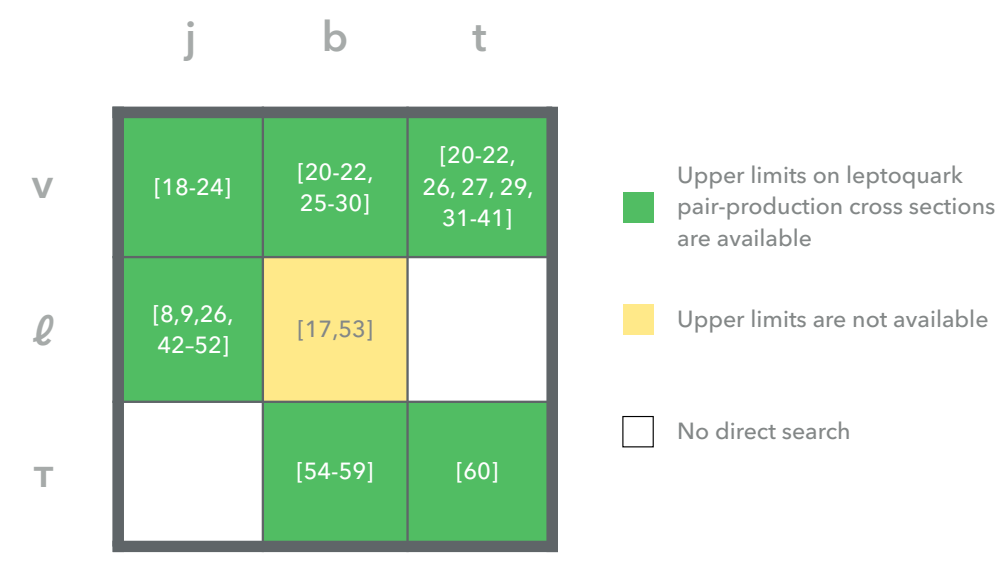

Figure 6. Summary of the current status of LQ searches at LHC after Runs 1 and 2. We include LQ searches and SUSY searches which have identical final states and decay topologies to LQ searches. Final states without any direct search are left white. The numbers give the references relevant to the various final states: $(\nu j)[18-24],(\nu b)$ [20-22, 25-30], $(\nu t)[20-22,26,27,29,31-41],(e j)$ and/or $(\mu j)[8,9,26,42-52],(e b)$ and $(\mu b)[17,53],(\tau b)$ [54-59], and $(\tau t)$ [60].

somewhat less satisfactory mass limits for specific leptoquark models in one case and no dedicated leptoquark (or equivalent RPV squark searches) in two cases. In all cases, we perform a recast of the bounds to obtain mass limits in our MLQ models. For the cases where experimenters provided full 95\% CL bounds on the cross section our MLQ model limits can be regarded with confidence. In the other cases we regard them as estimates for what the limits could have been.

We close this section with the LQ matrix with elements filled with references to experiments which performed searches corresponding to the LQ matrix final states. Green indicates that at least one experiment published the 95\% CL upper bound on the pair production cross section as a function of LQ mass.

\section{Minimal leptoquark models}

Here we define a set of MLQ models. The idea behind those models is simple. Each model has one leptoquark in a single $\mathrm{SU}(3)_{\text {color }} \times \mathrm{SU}(2)_{\text {weak }} \times \mathrm{U}(1)_{Y}$ representation coupling to only one SM lepton-quark fermion bilinear. Therefore to define the MLQ models we just need to identify all possible lepton-quark bilinears and their transformation properties under the SM gauge group. For each MLQ model we then obtain the lower bound on the mass of the MLQ by comparing the predicted cross section times branching fraction into LQ final states with the upper bound from experiment. To do so we need to determine the cross section times branching fraction into the possible symmetric finals states for each MLQ. It will be convenient to parameterize the answer as $\mu \times \sigma_{S}$ or $\mu \times \sigma_{V}$ for scalar or vector MLQs. Here $\sigma_{S}$ and $\sigma_{V}$ are the fiducial cross sections shown in figure 4 and $\mu$ is a number defined as

$$
\mu \equiv \frac{\sigma(p p \rightarrow S \bar{S})}{\sigma_{S}} \operatorname{Br}(S \bar{S} \rightarrow \text { final state }), \quad \mu \equiv \frac{\sigma(p p \rightarrow V \bar{V})}{\sigma_{V}} \operatorname{Br}(V \bar{V} \rightarrow \text { final state }) .
$$


$\mu$ may be greater than 1 when multiple LQs are produced and less when branching fractions into the final state are nontrivial.

To define the MLQ models, we identify the lepton-quark bilinears in the SM that we can couple to LQs. Ignoring the three generations for the moment, these are given by arbitrary quark or antiquark fields multiplying arbitrary lepton fields. Enumerating all the possibilities is easiest when all SM fermions are interpolated by left chirality fields. ${ }^{4}$ One generation of fermion fields is $Q, U^{c}, D^{c}, L, E^{c}, N^{c}$. Note that we have included possible singlet right-handed neutrinos. Scalar $S$ and vector $V$ leptoquarks couple to quark-lepton bilinears as

$$
S(\boldsymbol{Q} \boldsymbol{L}) \quad \text { and } \quad V^{\mu}\left(\boldsymbol{Q}^{\dagger} \bar{\sigma}_{\mu} \boldsymbol{L}\right)
$$

respectively. Here $\bar{\sigma}_{\mu}$ is a vector of $2 \times 2$ matrices which contracts the vector index of the LQ with the fermion spinors. This matrix appears in all vector LQ couplings to fermions. To avoid notational clutter we suppress it in subsequent formulas. $\boldsymbol{Q}$ and $\boldsymbol{L}$ can be any of the quark and lepton fields, i.e., $\boldsymbol{Q} \in\left\{Q, U^{c}, D^{c}\right\}$ and $\boldsymbol{L} \in\left\{L, E^{c}, N^{c}\right\}$. For both scalars and vectors there are $3 \times 3$ different possibilities (before considering generation number).

Adding in the three generations, each of the above described scalar or vector LQs we can couple to one of 3 generations of quarks and 3 generations of leptons for an additional multiplicity of 9 . Thus in total there are $3\left(Q, U^{c}, D^{c}\right) \times 3\left(L, E^{c}, N^{c}\right) \times 3$ (quark gen.) $\times$ 3 (lepton gen.) $\times 2(S$ or $V)=162$ different MLQ models. Note that we are defining the MLQ models in the mass eigenstate basis for the quarks and charged leptons. In doing so, we ignore the small mismatch between mass eigenstate bases for the doublet up- and downtype quark fields, this is equivalent to ignoring leptoquark decays which are proportional to $\lambda$ times small CKM matrix elements. The basis for neutrinos is irrelevant because the different neutrino flavors are indistinguishable final states.

For example, we can have a scalar leptoquark coupling as $\lambda S\left(Q_{1} E_{1}^{c}\right)$ where the subscripts are generation indices. This is a scalar which is an $\mathrm{SU}(2)_{\text {weak }}$ doublet. One of its components couples to up quarks and $e^{+}$and the other couples to down quarks and $e^{+}$. Thus this MLQ model actually contains two mass-degenerate scalar leptoquarks which means that the total cross section for leptoquark production is twice as large as $\sigma_{S}$. Each of the two components of $S$ couples to only one fermion bilinear. Therefore both decay with $100 \%$ branching fraction, one decays to $\left(e^{+} u\right)$, the other to $\left(e^{+} d\right)$. In terms of experimentally distinguishable final states both LQs decay to $\left(e^{+} j\right)$. Thus the total cross section times branching fraction to the final state $\left(e^{+} j\right)\left(e^{-} j\right)$ in this model is two times the scalar fiducial cross section in figure 6 , and the $\mu$-factor for this model is $\mu=2$. We show the pair production and decay $\mu$-factors of all MLQs in table 1 . Consider as another example the leptoquark coupling to $U_{3}^{c \dagger} E_{1}^{c}$. This is a vector leptoquark with a unique coupling and decay to top quarks and $e^{+}$. It is an $\mathrm{SU}(2)_{\text {weak }}$ singlet and therefore has no multiplicity. The cross section times branching to the final state $\left(e^{-} \bar{t}\right)\left(e^{+} t\right)$ is then equal to the fiducial cross section $\sigma_{V}$, and the $\mu$-factor is 1 . Note that the final state of this leptoquark is distinguishable from the final state of the scalar leptoquark coupling to $U_{3}^{c} E_{1}^{c}$ which decays

\footnotetext{
${ }^{4}$ To pass to this notation right-chirality fields are replaced by their (left-chirality) charge-conjugates $\psi_{R} \rightarrow \psi_{L}^{c} \equiv i \sigma_{2} \psi_{R}^{*}$. For more on the $Q U D L E N$ notation see appendix A.
} 
to $\left(e^{+} \bar{t}\right)$ giving the pair production final state $\left(e^{-} t\right)\left(e^{+} \bar{t}\right)$ which has the same final particles but different resonance pairs. Its $\mu$-factor is also 1 .

To get MLQs with non-trivial branching fractions we must consider scalar leptoquarks coupling to $Q L$ and vectors coupling to $Q^{\dagger} L$. In each case, there are two possibilities, the leptoquark can be isospin singlet or triplet.

Turning first to the singlet case, the scalar couples to $\epsilon_{\alpha \beta} Q_{\alpha} L_{\beta}$ while $\delta_{\alpha \beta} Q_{\alpha}^{\dagger} L_{\beta}$ is the vector coupling. ${ }^{5}$ Consider as an example the singlet LQ coupling to third generation quarks and first generation leptons. It couples to the fermion bilinear $\left(t e^{-}-b \nu\right)$. Thus there are 4 distinct final states

$$
S: \frac{1}{4}\left[\left(e^{-} t\right)\left(e^{+} \bar{t}\right)+b \bar{b} \mathbb{E}_{\mathrm{T}}+\left(e^{-} t\right) \bar{b} \mathbb{E}_{\mathrm{T}}+\left(e^{+} \bar{t}\right) b \mathbb{E}_{\mathrm{T}}\right],
$$

where the $1 / 4$ indicates that each final state has branching fraction $25 \%$. This is an example in which isospin predicts symmetric final states $\left(e^{-} t\right)\left(e^{+} \bar{t}\right)$ and $b \bar{b} \mathbb{E}_{\mathrm{T}}$ as well as asymmetric final states $\left(e^{-} t\right) \bar{b} \mathbb{E}_{\mathrm{T}},\left(e^{+} \bar{t}\right) b \mathbb{E}_{\mathrm{T}}$. Again, we focus only on the symmetric case because it does not have neutrinos and is usually more sensitive than the asymmetric one even though that has multiplicity of 2 . In the literature this case is sometimes called $\beta=1 / 2$ because the LQ has two different decays with branching fractions $B r \equiv \beta=1 / 2$.

An isospin singlet vector coupling to third generation quarks and first generation leptons couples to $\left(\bar{t} \nu+\bar{b} e^{-}\right)$and also gives rise to $25 \%$ branching fractions

$$
V: \frac{1}{4}\left[\left(e^{-\bar{b}}\right)\left(e^{+} b\right)+t \bar{t} \mathbb{E}_{\mathrm{T}}+\left(e^{-\bar{b}}\right) t \mathbb{E}_{\mathrm{T}}+\left(e^{+} b\right) \bar{t} \mathbb{E}_{\mathrm{T}}\right] .
$$

In both the scalar and the vector cases the branching fractions to any single final state are reduced to $25 \%$. There is no multiplicity factor from the cross section, thus both have $\mu=0.25$.

An isospin triplet MLQ model contains three LQs which each couples to a different combination of fermions. For example, choosing the MLQ with couplings to third generation quarks and first generation leptons, the couplings are to $\left(t \nu,\left(t e^{-}+b \nu\right) / \sqrt{2}, b e^{-}\right)$for the scalar case and $\left(\bar{t} e^{-},\left(\bar{t} \nu-\bar{b} e^{-}\right) / \sqrt{2}, \bar{b} \nu\right)$ for the vector case. Since each of the three LQs can be pair produced with the fiducial cross section one obtains the final states

$$
\begin{aligned}
& S: t \bar{t} \mathbb{E}_{\mathrm{T}}+\left(e^{-} b\right)\left(e^{+} \bar{b}\right)+\frac{1}{4}\left[\left(e^{-} t\right)\left(e^{+} \bar{t}\right)+b \bar{b} \mathbb{E}_{\mathrm{T}}+\left(e^{-} t\right) \bar{b} \mathbb{E}_{\mathrm{T}}+\left(e^{+} \bar{t}\right) b \mathbb{E}_{\mathrm{T}}\right], \\
& V:\left(e^{-} \bar{t}\right)\left(e^{+} t\right)+\bar{b} b \mathbb{E}_{\mathrm{T}}+\frac{1}{4}\left[\left(e^{-} \bar{b}\right)\left(e^{+} b\right)+t \bar{t} \mathbb{E}_{\mathrm{T}}+\left(e^{-} \bar{b}\right) t \mathbb{E}_{\mathrm{T}}+\left(e^{+} b\right) \bar{t} \mathbb{E}_{\mathrm{T}}\right] .
\end{aligned}
$$

Here the most promising search channels are the final states $\left(e^{-} b\right)\left(e^{+} \bar{b}\right)$ and $\left(e^{-} \bar{t}\right)\left(e^{+} t\right)$ which occur with $\mu$-factors 1 .

Finally, for isospin triplet MLQs coupling to quarks of the first or second generation the final state quarks produce indistinguishable light-jets. Thus here the final states are

$$
S \text { or } V: \frac{5}{4}\left[\left(e^{-} j\right)\left(e^{+} j\right)+j j \mathbb{E}_{\mathrm{T}}\right]+\frac{1}{4}\left[\left(e^{-} j\right) j \mathbb{E}_{\mathrm{T}}+\left(e^{+} j\right) j \mathbb{E}_{\mathrm{T}}\right] .
$$

yielding $\mu$-factors of 1.25 for both of the symmetric final states $\left(e^{-} j\right)\left(e^{+} j\right)$ and $j j \mathbb{E}_{\mathrm{T}}$.

$$
{ }^{5} \text { Here the } \epsilon=\left(\begin{array}{cc}
0 & 1 \\
-1 & 0
\end{array}\right) \text { and } \delta=\left(\begin{array}{ll}
1 & 0 \\
0 & 1
\end{array}\right) \text {. }
$$




\begin{tabular}{|ccccccccccc|}
\hline$p p \rightarrow S \bar{S} \rightarrow(\boldsymbol{l q})(\overline{\boldsymbol{l}} \overline{\boldsymbol{q}})$ & $Q L$ triplet & $Q L$ singlet & $U^{c} L$ & $D^{c} L$ & $Q E^{c}$ & $U^{c} E^{c}$ & $D^{c} E^{c}$ & $Q N^{c}$ & $U^{c} N^{c}$ & $D^{c} N^{c}$ \\
\hline$(\nu j)$ & 1.25 & 0.25 & 1 & 1 & $\times$ & $\times$ & $\times$ & 2 & 1 & 1 \\
$(\nu b)$ & 0.25 & 0.25 & $\times$ & 1 & $\times$ & $\times$ & $\times$ & 1 & $\times$ & 1 \\
$(\nu t)$ & 1 & $\times$ & 1 & $\times$ & $\times$ & $\times$ & $\times$ & 1 & 1 & $\times$ \\
$(e j),(\mu j),(\tau j)$ & 1.25 & 0.25 & 1 & 1 & 2 & 1 & 1 & $\times$ & $\times$ & $\times$ \\
$(e b),(\mu b),(\tau b)$ & 1 & $\times$ & $\times$ & 1 & 1 & $\times$ & 1 & $\times$ & $\times$ & $\times$ \\
$(e t),(\mu t),(\tau t)$ & 0.25 & 0.25 & 1 & $\times$ & 1 & 1 & $\times$ & $\times$ & $\times$ & $\times$ \\
\hline
\end{tabular}

\begin{tabular}{|cccccccccccc}
\hline$p p \rightarrow V \bar{V} \rightarrow(\boldsymbol{l q})(\overline{\boldsymbol{l}} \overline{\boldsymbol{q}})$ & $Q^{\dagger} L$ triplet & $Q^{\dagger} L$ singlet & $U^{c \dagger} L$ & $D^{c \dagger} L$ & $Q^{\dagger} E^{c}$ & $U^{c \dagger} E^{c}$ & $D^{c \dagger} E^{c}$ & $Q^{\dagger} N^{c}$ & $U^{c \dagger} N^{c}$ & $D^{c \dagger} N^{c}$ \\
\hline$(\nu j)$ & 1.25 & 0.25 & 1 & 1 & $\times$ & $\times$ & $\times$ & 2 & 1 & 1 \\
$(\nu b)$ & 1 & $\times$ & $\times$ & 1 & $\times$ & $\times$ & $\times$ & 1 & $\times$ & 1 \\
$(\nu t)$ & 0.25 & 0.25 & 1 & $\times$ & $\times$ & $\times$ & $\times$ & 1 & 1 & $\times$ \\
$(e j),(\mu j),(\tau j)$ & 1.25 & 0.25 & 1 & 1 & 2 & 1 & 1 & $\times$ & $\times$ \\
$(e b),(\mu b),(\tau b)$ & 0.25 & 0.25 & $\times$ & 1 & 1 & $\times$ & 1 & $\times$ & $\times$ \\
$(e t),(\mu t),(\tau t)$ & 1 & $\times$ & 1 & $\times$ & 1 & 1 & $\times$ & $\times$ & $\times$ \\
\end{tabular}

Table 1. (Upper table) $\mu$-factors for production and decay $\sigma(p p \rightarrow S \bar{S} \rightarrow(\boldsymbol{l q})(\overline{\boldsymbol{l}} \overline{\boldsymbol{q}}))$ in different scalar MLQ models. Rows correspond to different final states whereas columns correspond to different MLQ models. For example, the first row of the first column corresponds to pair production of a scalar LQ which decays to $(\nu j)$ via a coupling in which $Q$ and $L$ are contracted into an isospin triplet. For any final state $(\boldsymbol{q} \boldsymbol{l})(\boldsymbol{q} \boldsymbol{l})$, the $\mu$-factor is the factor which multiplies the fiducial cross section $\sigma_{S}$ in figure 4 to account for multiplicities and branching fractions. MLQs which couple to $\mathrm{SU}(2)_{\text {weak }}$-singlet fermions always have $\mu=1$. A " $\times$ " entry indicates that MLQ model does not contribute to the final state. (Lower table) $\mu$-factors for the vector leptoquark $V$. Comparing to the scalar case, differences exist only in the first two columns. Note that the $\mu$-factors for scalar MLQs decaying to $(\nu t),(e b),(\mu b),(\tau b)$ and vector MLQs $(\nu b),(e t),(\mu t),(\tau t)$ are equal to 1 irrespective of MLQ model.

We summarize all possible $\mu$-factors in table 1. For each final state, i.e. each row in the table, one can read off the $\mu$-factor for different MLQ models contributing to this final state. Alternatively, given a particular MLQ model defined by the MLQs' coupling to SM fermion bilinears $[\boldsymbol{L} \boldsymbol{Q}]$, the entries in the corresponding column show the $\mu$-factors for each attainable LQ matrix final state. For example, for $S$ coupling to $\left[Q_{3} L_{2}\right.$ triplet], the allowed final states are $(\nu b),(\nu t),(\mu b)$, and $(\mu t)$ with corresponding $\mu$-factors $0.25,1,1,0.25$. Thus the cross sections for $p p \rightarrow(\nu t)(\nu \bar{t})$ and $p p \rightarrow(\mu b)(\mu b)$ are 4 times larger than $p p \rightarrow(\nu b)(\nu b)$ or $p p \rightarrow(\mu t)(\mu \bar{t})$ and hence better suited for putting bounds on the mass of this MLQ.

To place bounds on all MLQ models with the same final state, it is important for the experimental collaborations to search for sufficiently high and low LQ masses. The lower masses are required to set bounds on MLQs with small cross sections whereas the highest masses are relevant for MLQs with large cross sections. The range in cross sections of MLQ models is bounded by $0.25 \times \sigma_{S}$ and $2 \times \sigma_{V}$. We illustrate this range as the cream band in figure 4 . Other models can in principle have even larger or smaller $\mu$ factors because of larger LQ multiplicities or smaller branching fractions to the final state under consideration. Thus a search mass window that is as large as possible is desirable. 

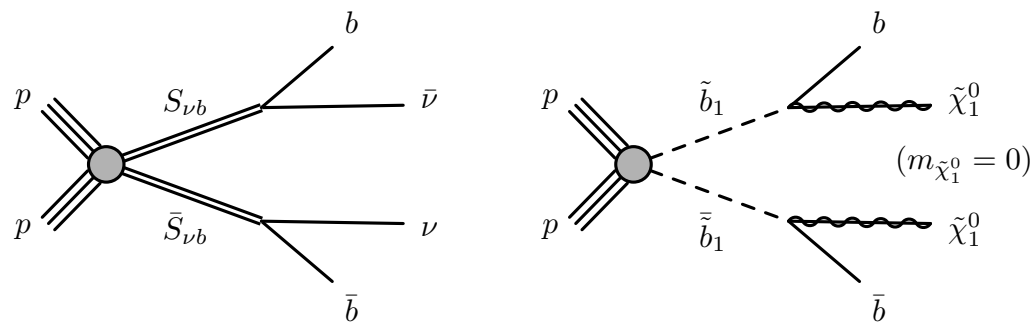

Figure 7. Diagrams for the process $p p \rightarrow S_{\nu b} \bar{S}_{\nu b} \rightarrow(b \bar{\nu})(\bar{b} \nu)$ (left) and the corresponding SUSY process $p p \rightarrow \tilde{b}_{1} \overline{\tilde{b}}_{1} \rightarrow\left(b \tilde{\chi}_{1}^{0}\right)\left(\bar{b} \tilde{\chi}_{1}^{0}\right)$ (right). For massless neutralinos the final-states and kinematic distributions of the two processes are identical. Similar identifications can be made for other $S_{\nu q}$ and squark searches. Here the "1" subscripts are the usual SUSY notation for lightest $b$-squark and lightest neutralino.

\section{Leptoquark searches organized by the LQ matrix}

LQs may be classified by their decay products and organized into the LQ matrix. The symmetric final states from pair-production where the LQ and its antiparticle decay in the same way give the strongest bounds. We therefore focus on the symmetric final states which can also be organized into the LQ matrix. The current status of searches for each final state is summarized in figure 6. The color of each entry of the matrix indicates whether a cross section bound as a function of mass has been made available; the numbers indicate relevant references. ${ }^{6}$ In the following subsections we review LQ searches organized by entries of the LQ matrix.

\section{$3.1 \nu j, \nu b$ and $\nu t$}

Searches for pair-produced scalar leptoquarks decaying into $(\nu j)(\nu j),(\nu b)(\nu b)$, and $(\nu t)(\nu t)$ final states are identical to those for pair-produced squarks $\tilde{q}_{1}[18-24]\left(\tilde{q}_{1}=\tilde{u}_{1}, \tilde{d}_{1}, \tilde{s}_{1}\right.$, or $\left.\tilde{c}_{1}\right), \tilde{b}_{1}[20-22,25-30]$, and $\tilde{t}_{1}[20-22,26,27,29,31-41]$, namely the pair-produced particles share the same spin, charge, decay final states and kinematics, if the neutralino $\tilde{\chi}_{1}^{0}$ is taken to be massless. An example is shown in figure 7 with the leptoquark production and decay diagram on the left and the corresponding squark diagram on the right. Thus constraints on squarks can be directly applied to $S_{\nu q}$ and vise visa. Such a translation has already been performed by ATLAS [26]. We obtain the strongest 95\% CL level limits by applying squark bounds from CMS Run 2 analyses, $m_{S_{\nu j}} \geq 1.05 \mathrm{TeV}[20,21], m_{S_{\nu b}} \geq 1.175 \mathrm{TeV}$ [21], and $m_{S_{\nu t}} \geq 1.07 \mathrm{TeV}[21]$.

The recast of the bounds to other MLQs is performed as follows. We digitize figure 7 in [21] for the observed 95\% CL upper limit on the light squark pair production cross section from searches at CMS, setting $m_{\tilde{\chi}_{1}^{0}}=0$. The digitized cross section limit is shown as the black solid curve in figure 8. The colored lines in the same plot show the theoretical cross sections of different MLQs that can decay into $(\nu j)(\nu j)$ final states. The curves are labeled with the cross sections given in terms of the $\mu$-factor times the relevant fiducial

\footnotetext{
${ }^{6}$ Searches in asymmetric final states $(\ell j)(\nu j)$ are given in $[44,45,47,51]$. Searches in asymmetric final states $(\tau t)(\nu b)$ and $(\tau b)(\nu t)$ are given in [61].
} 


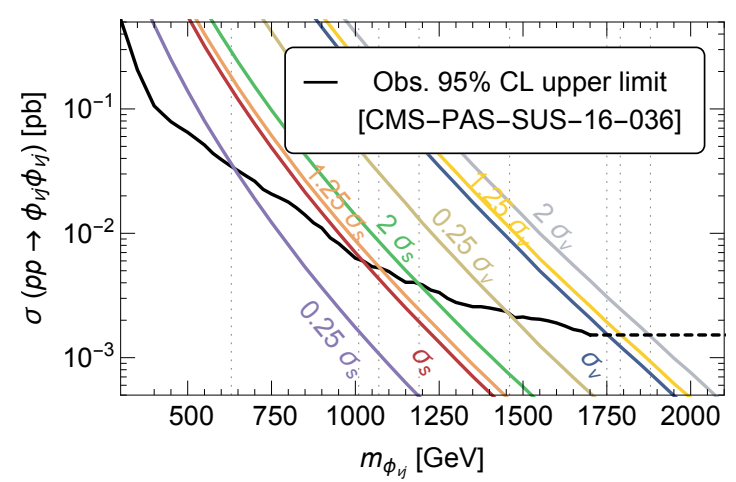

Figure 8. Bounds on scalar and vector MLQs which decay into $(\nu j)(\nu j)$ final states. The observed 95\% CL upper limit on the production cross section (black solid line) is taken from light squark searches at CMS [21]. The search stopped at $1.7 \mathrm{TeV}$, but we extrapolated the cross section limit by assuming that it becomes independent of LQ mass for large masses (black dashed line). The theoretical cross sections for different MLQs which can decay into $(\nu j)(\nu j)$ final states are shown as colored lines and labeled with their cross sections. The intersects (vertical dotted lines) correspond to lower limits on the MLQ masses.

cross section, $\sigma_{S}$ or $\sigma_{V}$, for scalar or vector LQs respectively. The intersects (dotted black lines) of the cross section curves with the experimental bound correspond to the lower mass bounds obtained in the different models. We obtain the bounds $630 \mathrm{GeV}$, $1 \mathrm{TeV}, 1.1 \mathrm{TeV}$, and $1.2 \mathrm{TeV}$ for scalar leptoquarks with $\mu$-factors of $0.25,1,1.25$, and 2 , respectively, and $1.45 \mathrm{TeV}$ for vector leptoquarks with $\mu$-factors of 0.25 . The CMS search stopped at $1.7 \mathrm{TeV}$ and therefore we cannot obtain reliable experimental bounds on heavier leptoquark candidates. To get an estimate, we extrapolated the experimental bound on the cross sections as independent of LQ mass for masses larger than $1.7 \mathrm{TeV}$ (shown as the black dotted line). This allows us to recast bounds on vector leptoquarks of $1.8 \mathrm{TeV}$, $1.8 \mathrm{TeV}$, and $1.9 \mathrm{TeV}$ for $\mu$-factors of $1,1.25$, and 2 respectively. Referring to the row labeled $S_{\nu j}$ and $V_{\nu j}$ in table 1 we can then associate the mass bounds corresponding to different $\mu$-factors to the different MLQ models. These bounds are summarized in table 2 , table 3, and table 4. Mass bounds in parenthesis are estimated bounds obtained using our extrapolated experimental cross section limits. Mass bounds without parenthesis are rigorous, they rely only on cross section bounds published by the experiments.

In addition ATLAS and CMS also looked for asymmetric decays into $(e j)(\nu j)$ or $(\mu j)(\nu j)$ final states. These searches require leptoquark decays to both $(e j)$ and $(\nu j)$ which is true for the $\mathrm{SU}(2)_{\text {weak }}$ singlet or triplet coupling to $Q L$ or $Q^{\dagger} L$. The latest $95 \%$ CL lower limit on the leptoquark mass from those channels is $m_{S_{\nu j}} \geq 850 \mathrm{GeV}$ [51]. This is comparable to the bound from searches for the symmetric final state $(e j)(e j)$ in the case of a singlet leptoquark coupling to $\left[Q_{1,2} L_{1,2,3}\right.$ singlet], but significantly weaker than the bound from the symmetric search for the triplet leptoquark coupling to $\left[Q_{1,2} L_{1,2,3}\right.$ triplet], see table 2 .

Also note that many searches for $(\nu j)(\nu j)$ final states and light squark searches did not veto $c$-jets. Therefore the limits also apply to $\phi_{\nu c}$. One might hope to improve the sensitivity of these searches to $\phi_{\nu c}$ with $c$-tagging. However, $c$-tagging efficiencies are cur- 


\begin{tabular}{|cc|c|c|c|c|}
\hline & & $U^{c} L, D^{c} L, U^{c} N^{c}, D^{c} N^{c}$ & $Q N^{c}$ & $Q L$ triplet & $Q L$ singlet \\
\hline \multirow{2}{*}{$S_{\nu j}$} & $\sigma_{\text {prod }}$ & $\times 1$ & $\times 2$ & $\times 1.25$ & $\times 0.25$ \\
& $m_{S_{\nu j}}$ & $\geq 1 \mathrm{TeV}[21]$ & $\geq 1.2 \mathrm{TeV}$ & $\geq 1.1 \mathrm{GeV}$ & $\geq 630 \mathrm{GeV}$ \\
\hline
\end{tabular}

\begin{tabular}{|cc|c|c|c|c|}
\hline & & $U^{c \dagger} L, D^{c \dagger} L, U^{c \dagger} N^{c}, D^{c \dagger} N^{c}$ & $Q^{\dagger} N^{c}$ & $Q^{\dagger} L$ triplet & $Q^{\dagger} L$ singlet \\
\hline \multirow{2}{*}{$V_{\nu j}$} & $\sigma_{\text {prod }}$ & $\times 1$ & $\times 2$ & $\times 1.25$ & $\times 0.25$ \\
& $m_{V_{\nu j}}$ & $(\geq 1.8 \mathrm{TeV})$ & $(\geq 1.9 \mathrm{TeV})$ & $(\geq 1.8 \mathrm{TeV})$ & $\geq 1.45 \mathrm{TeV}$ \\
\hline
\end{tabular}

Table 2. (Upper) 95\% CL lower limit on the mass of scalar MLQs $S_{\nu j}$ obtained from figure 8. The bounds apply to LQs coupling to the first or second generation quarks and neutrinos of any generation. (Lower) 95\% CL lower limit on the mass of vector MLQs $V_{\nu j}$ which decay into $(\nu j)(\nu j)$ final states. For bounds greater than $1.7 \mathrm{TeV}$, we have assumed that the experimental upper limit on the cross section is flat as the mass increases. Since these bounds are based on our assumption they cannot be considered solid and we marked them with parentheses. Note that [21] obtain a mass bound of $1050 \mathrm{GeV}$ while our plot figure 8 shows an intersect at $\sim 1 \mathrm{TeV}$, this small discrepancy may be a reflection of the inaccuracy of our digitization of the experimental cross section bound from figure 7 in [21] or a difference in assumed $K$-factor.

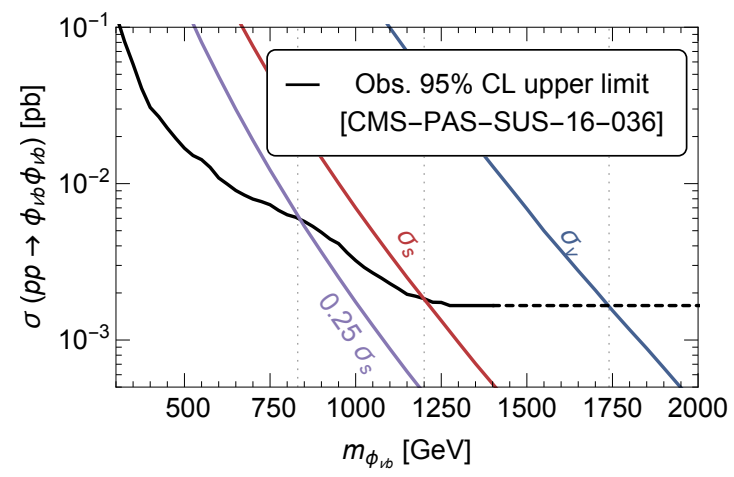

Figure 9. Bounds on scalar and vector MLQs that decay into $(\nu b)(\nu b)$ final states. The observed 95\% CL upper limit on the production cross section (black solid line) is taken from sbottom searches at CMS [21] which stopped at $1.4 \mathrm{TeV}$. Our analysis parallels that of figure 8.

\begin{tabular}{|cc|c|c|}
\hline & & $D^{c} L, Q N^{c}, D^{c} N^{c}$ & $(Q L$ triplet $), Q L$ singlet \\
\hline \multirow{3}{*}{$S_{\nu b}$} & $\sigma_{\text {prod }}$ & $\times 1$ & $\times 0.25$ \\
& $m_{S_{\nu b}}$ & $\geq 1.2 \mathrm{TeV}[21]$ & $\geq 830 \mathrm{GeV}$ \\
\hline
\end{tabular}

\begin{tabular}{|cc|c|}
\hline & & $D^{c \dagger} L, Q^{\dagger} N^{c}, D^{c \dagger} N^{c},\left(Q^{\dagger} L\right.$ triplet $)$ \\
\hline \multirow{2}{*}{$V_{\nu b}$} & $\sigma_{\text {prod }}$ & $\times 1$ \\
& $m_{V_{\nu b}}$ & $(\geq 1.7 \mathrm{TeV})$ \\
\hline
\end{tabular}

Table 3. $95 \%$ CL lower limits on the masses of MLQs which decay into $(\nu b)(\nu b)$ final states for the scalar LQs (left) and vector MLQs (right).

\begin{tabular}{|cc|c|}
\hline & & $U^{c} L, Q N^{c}, U^{c} N^{c},(Q L$ triplet $)$ \\
\hline \multirow{3}{*}{$S_{\nu t}$} & $\sigma_{\text {prod }}$ & $\times 1$ \\
& $m_{S_{\nu t}}$ & $\geq 1.1 \mathrm{TeV}[21]$ \\
\hline
\end{tabular}

\begin{tabular}{|cc|c|c|}
\hline & & $U^{c \dagger} L, Q^{\dagger} N^{c}, U^{c \dagger} N^{c}$ & $Q L$ triplet, $Q L$ singlet \\
\hline \multirow{2}{*}{$V_{\nu t}$} & $\sigma_{\text {prod }}$ & $\times 1$ & $\times 0.25$ \\
& $m_{V_{\nu t}}$ & $(\geq 1.7 \mathrm{TeV})$ & $(\geq 1.4 \mathrm{TeV})$ \\
\hline
\end{tabular}

Table 4. $95 \%$ CL lower limits on the masses of MLQs which decay into $(\nu t)(\nu t)$ final states for scalar LQs (left) and vector LQs (right). 


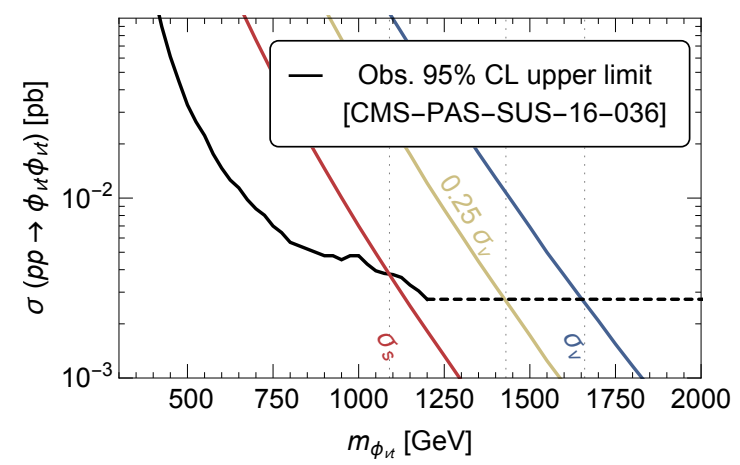

Figure 10. Bounds on scalar and vector MLQs which decay into $(\nu t)(\nu t)$ final states. The observed 95\% CL upper limit on the production cross section (black solid line) is taken from stop searches at CMS [21] which stopped at $1.2 \mathrm{TeV}$. Our analysis parallels that of figure 8.

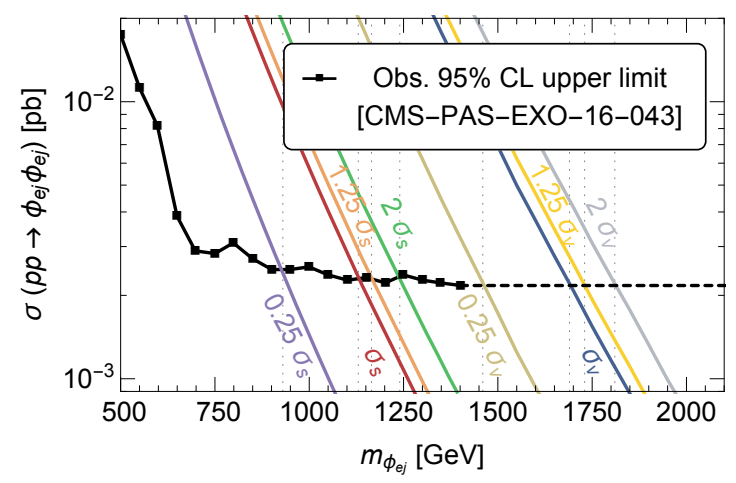

Figure 11. Bounds on scalar and vector leptoquarks that decay into $(e j)(e j)$ final states. The observed 95\% CL upper limit on the production cross section (black solid line) is taken from a search for leptoquarks in the $(e j)(e j)$ final state by CMS [52] which stopped at $1.4 \mathrm{TeV}$. See the caption of figure 8 for details.

rently too small for this to be effective. For example, ref. [23] $\left(8 \mathrm{TeV}, 20 \mathrm{fb}^{-1}\right)$ conducted a $\tilde{c}_{1}$ search with efficiencies: $c$-tag $19 \%, b$-mistagged-as- $c 13 \%$, and $j$-mistagged-as- $c 0.5 \%$. They obtained the bound $m_{\tilde{c}_{1}\left(S_{\nu c}\right)} \geq 540 \mathrm{GeV}$ which is weaker than the bound from the flavor inclusive analysis with the same data set [19], $m_{\tilde{c}_{1}\left(S_{\nu c}\right)} \geq 580 \mathrm{GeV}$. Efficiencies of $c$-tagging did not improve significantly in Run 2 [62].

\section{$3.2 e j$ and $\mu j$}

Leptoquarks decaying into $(e j)(e j)$ and $(\mu j)(\mu j)$ have been a focus of LHC leptoquark searches with both Run 1 and Run 2 data [8, 9, 26, 42-52]. No significant signal has been discovered, and 95\% CL lower limits on leptoquark masses are: $m_{S_{e j}} \geq 1.13 \mathrm{TeV}$ [52] and $m_{S_{\mu j}} \geq 1.17 \mathrm{GeV}[9]$. We summarize these bounds and our recasts from the cross section plots figure 5 and figure 11 in table 5 . Note that there is a stronger limit on $m_{S_{e j}} \geq 1.73 \mathrm{TeV}$ from single leptoquark production [63]. However this limit assumes a large coupling $(\lambda \geq 1)$ between up quarks and the leptoquark. For smaller couplings the single leptoquark cross section becomes very small and no limit can be obtained. 


\begin{tabular}{|cc|c|c|c|c|}
\hline & & $U^{c} L, D^{c} L, U^{c} E^{c}, D^{c} E^{c}$ & $Q E^{c}$ & $(Q L$ triplet $)$ & $(Q L$ singlet $)$ \\
\hline \multirow{2}{*}{$S_{e j}$} & $\sigma_{\text {prod }}$ & $\times 1$ & $\times 2$ & $\times 1.25$ & $\times 0.25$ \\
& $m_{S_{e j}}$ & $\geq 1.13 \mathrm{TeV}[52]$ & $\geq 1.2 \mathrm{TeV}$ & $\geq 1.2 \mathrm{TeV}$ & $\geq 930 \mathrm{GeV}$ \\
\hline \multirow{2}{*}{$S_{\mu j}$} & $\sigma_{\text {prod }}$ & $\times 1$ & $\times 2$ & $\times 1.25$ & $\times 0.25$ \\
& $m_{S_{\mu j}}$ & $\geq 1.17 \mathrm{TeV}[9]$ & $\geq 1.3 \mathrm{TeV}$ & $\geq 1.2 \mathrm{TeV}$ & $\geq 950 \mathrm{GeV}$ \\
\hline
\end{tabular}

\begin{tabular}{|cc|c|c|c|c|}
\hline & & $U^{c \dagger} L, D^{c \dagger} L, U^{c \dagger} E^{c}, D^{c \dagger} E^{c}$ & $Q^{\dagger} E^{c}$ & $Q^{\dagger} L$ triplet & $Q^{\dagger} L$ singlet \\
\hline \multirow{2}{*}{$V_{\text {ej }}$} & $\sigma_{\text {prod }}$ & $\times 1$ & $\times 2$ & $\times 1.25$ & $\times 0.25$ \\
& $m_{V_{e j}}$ & $(\geq 1.7 \mathrm{TeV})$ & $(\geq 1.8 \mathrm{TeV})$ & $(\geq 1.7 \mathrm{TeV})$ & $(\geq 1.5 \mathrm{TeV})$ \\
\hline \multirow{2}{*}{$V_{\mu j}$} & $\sigma_{\text {prod }}$ & $\times 1$ & $\times 2$ & $\times 1.25$ & $\times 0.25$ \\
& $m_{V_{\mu j}}$ & $(\geq 1.7 \mathrm{TeV})$ & $(\geq 1.9 \mathrm{TeV})$ & $(\geq 1.8 \mathrm{TeV})$ & $(\geq 1.5 \mathrm{TeV})$ \\
\hline
\end{tabular}

Table 5. (Upper table) 95\% CL lower limits on the masses of the scalar MLQs $S_{e j}$ and $S_{\mu j}$. The mass bounds in the first column are from [52] and [9] while the mass bounds in the remaining columns are our recasts, which use our $\mu$-factors and the experimental bounds on the production cross section from [52] and [9] respectively. The SM fermions involved in the couplings are the firstor second-generation quarks and electrons or muons. (Lower table) 95\% CL recast lower limits on the masses of vector leptoquarks $V_{e j}$ and $V_{\mu j}$.

\section{$3.3 e b$ and $\mu b$}

Many searches for $(e j)(e j)$ or $(\mu j)(\mu j)$ final states did not veto heavy flavors and their bounds apply to $(e b)(e b)$ or $(\mu b)(\mu b)$ as well. However, one can improve the sensitivity of the searches with $b$-tagging.

SUSY with RPV allows a superpotential coupling $\hat{L} \hat{Q} \hat{D}^{c}$ for the superfields. This superpotential coupling gives rise to a Yukawa coupling $\tilde{d}^{c \dagger} Q L$ in which the $\tilde{d}^{c}$ squark couples like a leptoquark. The final-state signature and kinematic distributions of pairproduced squarks which decay via this Yukawa coupling are identical to production and decay of scalar leptoquarks (e.g., see figure 12). Such a one-to-one correspondence to RPV squarks exists for all the leptoquarks coupling to $[L Q]$ or $\left[L D^{c}\right]$. ATLAS have conducted this type of RPV stop search with Run 1 [53] and Run 2 [17] data. The process searched for is $p p \rightarrow \tilde{t}_{1} \tilde{t}_{1}$ where $\tilde{t}_{1}$ decays into the final states $(e b)$ or $(\mu b)$ with at least one $b$-tag required in [17]. No excess was found for the stop masses searched. We take the strongest bounds from [17] $\left(13 \mathrm{TeV}, 36 \mathrm{fb}^{-1}\right)$ at the special points where the decays are $100 \%$ to $(e b)$ or $100 \%$ to $(\mu b)$. These can be directly reinterpreted as bounds on a scalar leptoquark decaying to $(\mu b)$ or $(e b)$. $S_{e b}$ and $S_{\mu b}$ are excluded up to $1.5 \mathrm{TeV}$ and $1.4 \mathrm{TeV}$ with $95 \%$ $\mathrm{CL}$, respectively. Assuming that the bounds on the cross section times branching fraction to the signal regions do not depend on the LQ mass for even heavier LQs we can recast these bounds to vector MLQs (see the right panel in figure 5 for the cross section bound plot for $(\mu b)$ and figure 13 for $(e b))$. We obtain the estimated bounds shown in parenthesis in table 6 . 

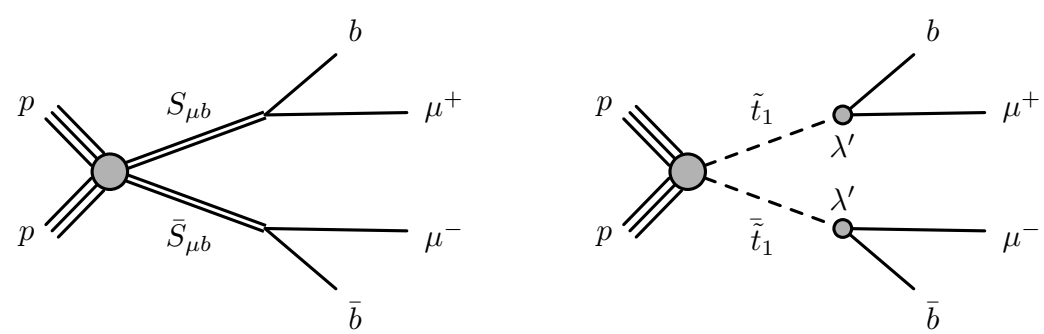

Figure 12. Diagrams for $p p \rightarrow S_{\mu b} \bar{S}_{\mu b} \rightarrow\left(b \mu^{+}\right)\left(\bar{b} \mu^{-}\right)(l e f t)$ and $p p \rightarrow \tilde{t}_{1} \overline{\tilde{t}}_{1} \rightarrow\left(b \mu^{+}\right)\left(\bar{b} \mu^{-}\right)(r i g h t)$. Final states and kinematic distributions of the two processes are identical.

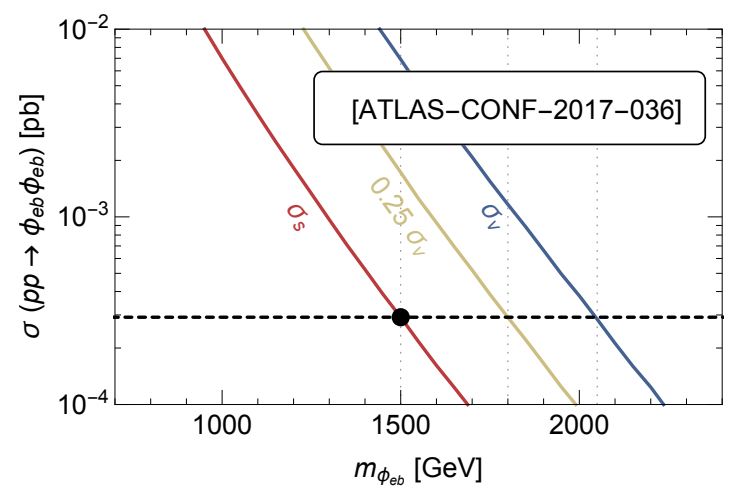

Figure 13. Bounds on scalar and vector MLQs which decay into $(e b)(e b)$ final states. The $95 \%$ CL on the stop/leptoquark mass (black dot) is provided in [17]. The remainder of the analysis is analogous to the right plot of figure 5 .

\begin{tabular}{|cc|c|}
\hline & & $D^{c} L, D^{c} E^{c}, Q E^{c},(Q L$ triplet $)$ \\
\hline \multirow{3}{*}{$S_{\text {eb }}$} & $\sigma_{\text {prod }}$ & $\times 1$ \\
& $m_{S_{e b}}$ & $\geq 1.5 \mathrm{TeV}[17]$ \\
\hline \multirow{2}{*}{$S_{\mu b}$} & $\sigma_{\text {prod }}$ & $\times 1$ \\
& $m_{S_{\mu b}}$ & $\geq 1.4 \mathrm{TeV}[17]$ \\
\hline
\end{tabular}

\begin{tabular}{|cc|c|c|}
\hline & & $D^{c \dagger} L, D^{c \dagger} E^{c}, Q^{\dagger} E^{c}$ & $Q^{\dagger} L$ triplet, $Q^{\dagger} L$ singlet \\
\hline \multirow{2}{*}{$V_{\text {eb }}$} & $\sigma_{\text {prod }}$ & $\times 1$ & $\times 0.25$ \\
& $m_{V_{e b}}$ & $(\geq 2.1 \mathrm{TeV})$ & $(\geq 1.8 \mathrm{TeV})$ \\
\hline \multirow{2}{*}{$V_{\mu b}$} & $\sigma_{\text {prod }}$ & $\times 1$ & $\times 0.25$ \\
& $m_{V_{\mu b}}$ & $(\geq 1.9 \mathrm{TeV})$ & $(\geq 1.7 \mathrm{TeV})$ \\
\hline
\end{tabular}

Table 6. (Left) $95 \%$ CL lower limits on the masses of scalar LQs $S_{e b}$ and $S_{\mu b}$. Both occur only with $\mu$-factors equal to one so that no recasts are necessary. The limits are adopted from RPV stop searches from ATLAS [17] (see figure 13 and figure 5). (Right) Estimated 95\% CL recast lower limits on the masses of vector LQs $V_{e b}$ and $V_{\mu b}$.

\section{4 et and $\mu t$}

Decays of leptoquarks into $(e t)(e t)$ and $(\mu t)(\mu t)$ give rise to several different final states depending on the subsequent top decays. We may have fully hadronic decays, semi-leptonic decays, and dileptonic decays:

$$
p p \rightarrow \phi_{\ell t} \bar{\phi}_{\ell t} \rightarrow \begin{cases}\left(t_{h} \ell^{+}\right)\left(\bar{t}_{h} \ell^{-}\right) & {[45.7 \%]} \\ \left(t_{l} \ell^{+}\right)\left(\bar{t}_{h} \ell^{-}\right)+\left(t_{h} \ell^{+}\right)\left(\bar{t}_{l} \ell^{-}\right) & {[43.8 \%]} \\ \left(t_{l} \ell^{+}\right)\left(\bar{t}_{l} \ell^{-}\right) & {[10.5 \%]}\end{cases}
$$




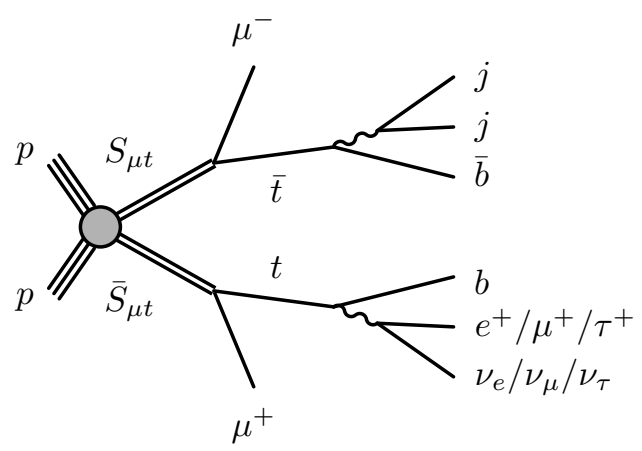

Figure 14. Diagram for $p p \rightarrow S_{\mu t} \bar{S}_{\mu t} \rightarrow\left(t_{l} \mu^{+}\right)\left(\bar{t}_{h} \mu^{-}\right)$. Similar final states and kinematics occur in RPV SUSY models.

where subscript $h$ or $l$ of $t$ indicates the top quark decays hadronically or leptonically, respectively. The first two cases of eq. (3.1) are preferable searches because of their large branching fractions (numbers in the brackets). Subsequent $\tau$-decays can further increase the number of different final states.

No dedicated searches for leptoquarks in any of these channels have been performed. However, the final states of $(e t)(e t)$ or $(\mu t)(\mu t)$ feature high jet-multiplicities, multiple electrons and/or muons, large missing energy, and multiple $b$ quarks. These features are also looked for in many SUSY and Exotics searches. Hence we can recast existing SUSY searches to set lower limits on the masses of $\phi_{e t}$ or $\phi_{\mu t}$.

Here we focus on the semi-leptonic case (eq. (3.1)b , see figure 14). CMS [64] presented a $13 \mathrm{TeV}$ stop search with $35.9 \mathrm{fb}^{-1}$ of data with 50 distinct signal regions. The final states can have multiple $b$-tags, triple leptons, and large missing energy. We generated signals with MadGraph5 aMC@NLO 2.5.4 [65] at leading order parton level. We considered leptoquark masses ranging from $500 \mathrm{GeV}$ to $1.6 \mathrm{TeV}$ with $100 \mathrm{GeV}$ steps, and applied the NLO cross sections from figure 4 . The signal can be triggered by two sets of dilepton triggers. The first set requires the loose isolation $\left(I_{\mathrm{mini}, \ell}<0.4\right)$ of two light leptons, with transverse momentum $p_{\mathrm{T}}$ satisfying $p_{\mathrm{T} \ell_{1}}, p_{\mathrm{T} \mu_{2}\left(e_{2}\right)}>23,8(12) \mathrm{GeV}$. The second set has no isolation requirement. It requires leptons with $p_{\mathrm{T} \ell_{1,2}}>8 \mathrm{GeV}$ and $H_{\mathrm{T}}^{30} \geq 300 \mathrm{GeV}$. The jets are clustered with the anti- $k_{\mathrm{T}}$ algorithm with $R=0.4$. The reconstructed jets are required to have $p_{\mathrm{T}} \geq 30 \mathrm{GeV}$ and pseudo-rapidity $|\eta|<2.4$. Reconstructed leptons are required to be well-isolated. They need to satisfy $I_{\text {mini }, \mu(e)}<0.16(0.12)$ together with $p_{\mathrm{T} \mu(e)}^{\text {ratio }}>0.69(0.76)$ or $p_{\mathrm{T} \mu(e)}^{\mathrm{rel}}>6.0(7.2) \mathrm{GeV}$. Besides isolation, the analysis requires the lepton pseudo-rapidity $\left|\eta_{\mu(e)}\right|<2.4(2.5)$ and the $p_{\mathrm{T}}$ of the leading three leptons $p_{\mathrm{T} \ell_{1}}, p_{\mathrm{T} \mu_{2}\left(e_{2}\right)}, p_{\mathrm{T} \ell_{3}} \geq 25,10(15), 10 \mathrm{GeV}$ if $H_{\mathrm{T}}^{30}<300 \mathrm{GeV}$ or $p_{\mathrm{T} \mu_{1}\left(e_{1}\right)}, p_{\mathrm{T} \mu_{2}\left(e_{2}\right)}, p_{\mathrm{T} \ell_{3}} \geq$ $10(15), 10(15), 10 \mathrm{GeV}$ if $H_{\mathrm{T}}^{30}>300 \mathrm{GeV}$. The identification efficiencies for muons (elec- 


\begin{tabular}{|c|c|c|c|c|c|}
\hline & \multicolumn{5}{|c|}{ Baseline cuts } \\
\hline$N_{\text {sel. } \ell}$ & & & $\geq 3$ & & \\
\hline$N_{\text {sel. jets }}$ & & & $\geq 2$ & & \\
\hline $\mathbb{E}_{\mathrm{T}}[\mathrm{GeV}]$ & & & $>50$ & & \\
\hline$m_{\ell \ell}[\mathrm{GeV}]$ & & & $\geq 12$ & & \\
\hline & SR5 & SR6 & SR9 & SR10 & SR16a \\
\hline$b$-tag & 1 & 1 & 2 & 2 & inclusive \\
\hline$H_{\mathrm{T}}[\mathrm{GeV}]$ & $60-400$ & $60-400$ & $60-400$ & $60-400$ & $\geq 60$ \\
\hline $\mathbb{E}_{\mathrm{T}}[\mathrm{GeV}]$ & $50-150$ & $150-300$ & $50-150$ & $150-300$ & $\geq 300$ \\
\hline$M_{\mathrm{T}}^{\min }[\mathrm{GeV}]$ & inclusive & inclusive & inclusive & inclusive & $<120$ \\
\hline Expected & $202 \pm 4 \pm 44$ & $25.6 \pm 1.9 \pm 4.6$ & $47.7 \pm 2.8 \pm 7.6$ & $5.3 \pm 0.5 \pm 0.6$ & $12.1 \pm 1.5 \pm 1.9$ \\
\hline Observed & 191 & 25 & 51 & 5 & 7 \\
\hline
\end{tabular}

Table 7. Summary of baseline selection cuts and selection cuts for the off- $Z$ signal regions (SR) that are most sensitive to $(e t)(e t)$ or $(\mu t)(\mu t)$ [64]. We also list the observed and expected number of events for each SR. See text for more details.

trons) are taken to be $96 \%$ (90\%). Kinematic variables used in the analysis are defined as:

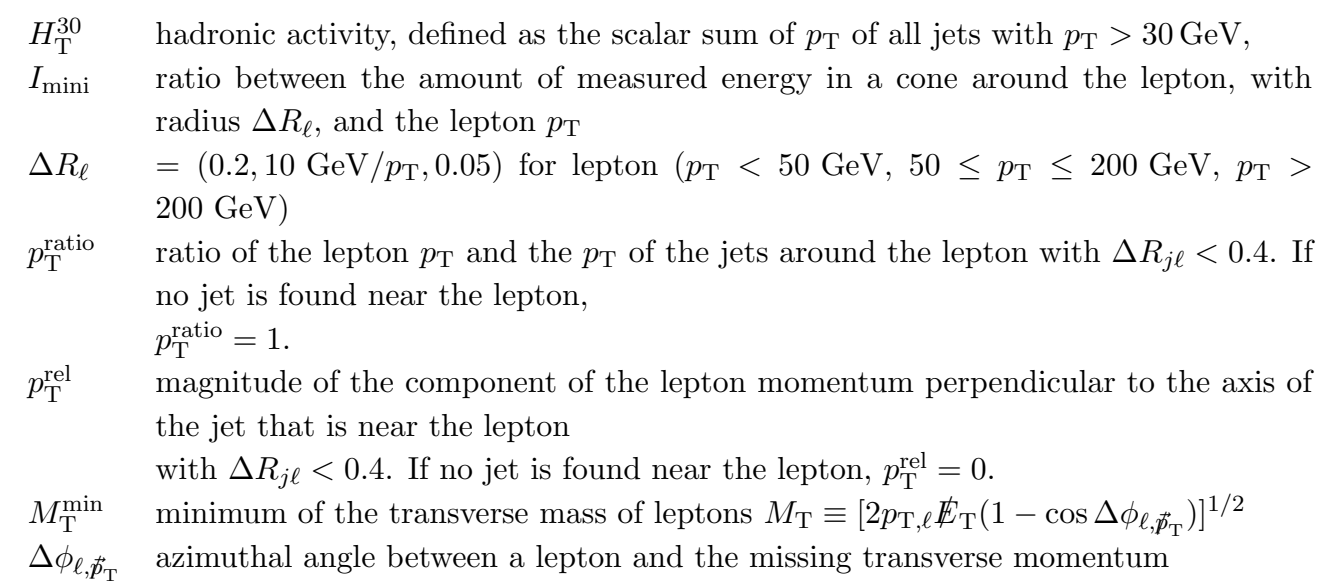

After the trigger and jet and lepton reconstructions, we pass the events through the baseline selection and divide them into the 25 off- $Z$ signal regions (SR's) and super signal regions (SSR's). $b$-tagging is performed on jets with $p_{\mathrm{T}} \geq 25 \mathrm{GeV}$ and $|\eta|<2.4$. The $b$-tagging, $c$ miss-tagging, and light-jet miss-tagging rates are $(70 \%, 10 \%, 1 \%)$. We then compare the number of remaining signal events with the number of the observed events and expected background given in [64]. Signal regions SR5, SR6, SR9, SR10, and SR16a are most sensitive to leptoquarks. Cuts for these regions, together with baseline cuts, are summarized in table 7 .

Using the central value of the background estimation in table 7 and assuming Poisson statistics, the resulting $95 \%$ CL lower limit on the mass of $S_{\mu t}$ is $800 \mathrm{GeV}$ from SR5 and SR9 and $700 \mathrm{GeV}$ from SR6, SR10, and SR16a. The resulting constraint on the mass of $S_{e t}$ is $900 \mathrm{GeV}$ from SR16a, $800 \mathrm{GeV}$ from SR10 and $700 \mathrm{GeV}$ from SR5, SR6, SR9. A common feature of the most sensitive search regions is that they allow relatively low hadronic activity. Note that our recast, using a simple cut and count search, is not optimized. Better sensitivity could be reached with an analysis that reconstructs at least one of the 


\begin{tabular}{|cc|c|c|}
\hline & & $U^{c} L, U^{c} E^{c}, Q E^{c}$ & $(Q L$ triplet $),(Q L$ singlet $)$ \\
\hline \multirow{2}{*}{$S_{\text {et }}$} & $\sigma_{\text {prod }}$ & $\times 1$ & $\times 0.25$ \\
& $m_{S_{\text {et }}}$ & $\geq 900 \mathrm{GeV}$ & $\geq 600 \mathrm{GeV}$ \\
\hline \multirow{2}{*}{$S_{\mu t}$} & $\sigma_{\text {prod }}$ & $\times 1$ & $\times 0.25$ \\
& $m_{S_{\mu t}}$ & $\geq 800 \mathrm{GeV}$ & $\geq 600 \mathrm{GeV}$ \\
\hline
\end{tabular}

\begin{tabular}{|cc|c|}
\hline & & $U^{c \dagger} L, U^{c \dagger} E^{c}, Q^{\dagger} E^{c},\left(Q^{\dagger} L\right.$ triplet $)$ \\
\hline \multirow{2}{*}{$V_{\text {et }}$} & $\sigma_{\text {prod }}$ & $\times 1$ \\
& $m_{V_{\text {et }}}$ & $\geq 1.5 \mathrm{TeV}$ \\
\hline \multirow{2}{*}{$V_{\mu t}$} & $\sigma_{\text {prod }}$ & $\times 1$ \\
& $m_{V_{\mu t}}$ & $\geq 1.3 \mathrm{TeV}$ \\
\hline
\end{tabular}

Table 8. $95 \%$ CL lower limits on the mass of MLQs which decay into $(e t)(e t)$ or $(\mu t)(\mu t)$ final states for the scalar leptoquarks (left) and the vector leptoquarks (right) from recasting the CMS SUSY search [64].

resonances of the signal. In table 8, we show the bounds on LQs decaying into $(e t)(e t)$ or $(\mu t)(\mu t)$ which we obtained for other MLQ models.

\section{$3.5 \tau j$}

In analogy with the $(e t)(e t)$ or $(\mu t)(\mu t)$ cases, the $\tau$ 's in $(\tau j)(\tau j)$ final states can be either hadronic or leptonic. The branching fractions for $\phi_{\tau j} \bar{\phi}_{\tau j}$ decays are given by

$$
p p \rightarrow \phi_{\tau j} \bar{\phi}_{\tau j} \rightarrow \begin{cases}\left(\tau_{h} j\right)\left(\tau_{h} j\right) & {[42.0 \%]} \\ \left(\tau_{l}^{+} j\right)\left(\tau_{h} j\right)+\left(\tau_{l}^{-} j\right)\left(\tau_{h} j\right) & {[45.6 \%]} \\ \left(\tau_{l}^{+} j\right)\left(\tau_{l}^{-} j\right) & {[12.4 \%]}\end{cases}
$$

We are not aware of any dedicated searches for these final states. However, three types of existing searches can be relevant: (1) leptoquarks which decay to $(\tau b)(\tau b)$ final states are intensively studied by ATLAS and CMS (see section 3.6). An analysis without imposing the $b$-tags would cover the $(\tau j)(\tau j)$ channel; $(2)$ searches for right-handed $W_{R}$ and righthanded neutrinos $N$ using the process $p p \rightarrow W_{R} \rightarrow \tau N \rightarrow \tau(\tau q \bar{q})$ has been conducted by $[58,59]$. The final particles of this process are identical to $\phi_{\tau j} \bar{\phi}_{\tau j}$. To apply this search one would have to adjust the signal kinematics and re-analyze the data; (3) our LQ final state can be also be covered by SUSY or Exotics searches which look for large missing energy with high $p_{\mathrm{T}}$-jets and zero, one, or two opposite-sign leptons.

Given the similarity of this final state with the well-covered $(\tau b)(\tau b)$ final state and given that SM backgrounds are small at large invariant masses we expect that a designated search will obtain bounds on leptoquark masses which are comparable to those for the $(\tau b)(\tau b)$ case. We have not been able to obtain reliable bounds for any of the MLQs in this final state and therefore left the corresponding entries in table 9 blank.

\section{$3.6 \tau b$}

LQ decays into the $(\tau b)(\tau b)$ final state have been well-covered by LHC searches for LQs coupling to third generation quarks and leptons with Run 1 and Run 2 data [54-59]. The latest $95 \%$ upper limit on the only scalar MLQ with this final state is $850 \mathrm{GeV}$ [59]. We recast the bound for the vector MLQs in table 10.

\section{$3.7 \tau t$}

$(\tau t)(\tau t)$ has the most complicated final states of the nine types of LQs in the LQ matrix because each of the four decay particles, $\tau^{+}, \tau^{-}, t$ or $\bar{t}$, can decay leptonically or hadronically. 


\begin{tabular}{|cc|c|c|c|c|}
\hline & & $U^{c} L, D^{c} L, U^{c} E^{c}, D^{c} E^{c}$ & $Q E^{c}$ & $Q L$ triplet & $Q L$ singlet \\
\hline \multirow{2}{*}{$S_{\tau j}$} & $\begin{array}{c}\sigma_{\text {prod }} \\
m_{S_{\tau j}}\end{array}$ & $\times 1$ & $\times 2$ & $\times 1.25$ & $\times 0.25$ \\
& & & & \\
\hline
\end{tabular}

\begin{tabular}{|cc|c|c|c|c|}
\hline & $U^{c \dagger} L, D^{c \dagger} L, U^{c \dagger} E^{c}, D^{c \dagger} E^{c}$ & $Q^{\dagger} E^{c}$ & $Q^{\dagger} L$ triplet & $Q^{\dagger} L$ singlet \\
\hline$V_{\tau j}$ & $\begin{array}{c}\sigma_{\text {prod }} \\
m_{V_{\tau j}}\end{array}$ & $\times 1$ & $\times 2$ & $\times 1.25$ & $\times 0.25$ \\
\hline
\end{tabular}

Table 9. MLQ models which decay into the final state $(\tau j)(\tau j)$. We have not been able to find searches which can reliably and easily be recast to obtain mass limits for these models.

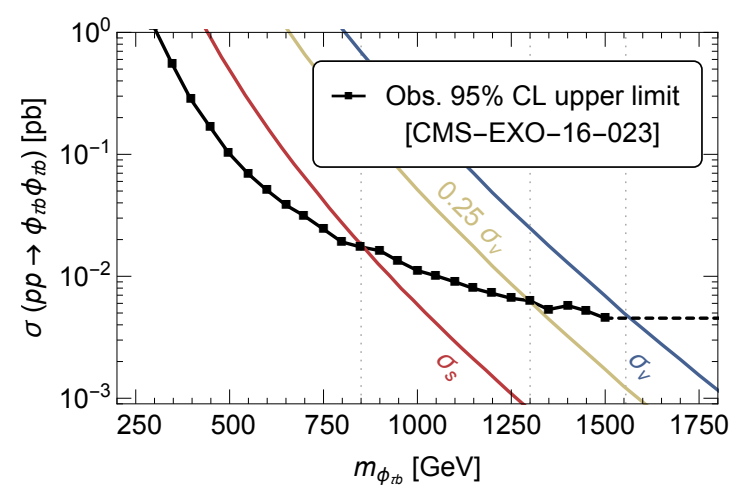

Figure 15. Bounds on scalar and vector MLQs which decay into $(\tau b)(\tau b)$ final states. The observed 95\% CL upper limit on the production cross section times branching fraction (black solid line) is taken from searches for LQs in this final state at CMS [59] that stopped at $1.5 \mathrm{TeV}$. The remainder of the analysis is analogous to figure 8.

\begin{tabular}{|cc|c|}
\hline & & $D^{c} L, D^{c} E^{c}, Q E^{c},(Q L$ triplet $)$ \\
\hline \multirow{3}{*}{$S_{\tau b}$} & $\sigma_{\text {prod }}$ & $\times 1$ \\
& $m_{S_{\tau b}}$ & $\geq 850 \mathrm{GeV}[59]$ \\
\hline
\end{tabular}

\begin{tabular}{|cc|c|c|}
\hline & & $D^{c \dagger} L, D^{c \dagger} E^{c}, Q^{\dagger} E^{c}$ & $Q^{\dagger} L$ triplet, $Q^{\dagger} L$ singlet \\
\hline \multirow{2}{*}{$V_{\tau b}$} & $\sigma_{\text {prod }}$ & $\times 1$ & $\times 0.25$ \\
& $m_{V_{\tau b}}$ & $(\geq 1.6 \mathrm{TeV})$ & $\geq 1.3 \mathrm{TeV}$ \\
\hline
\end{tabular}

Table 10. 95\% CL lower limits on the mass of MLQs which decay into $(\tau b)(\tau b)$ final states for the scalar leptoquarks (left) and the vector leptoquarks (right).

The multiplicity of possible final states, neglecting charge differences, is 10 .

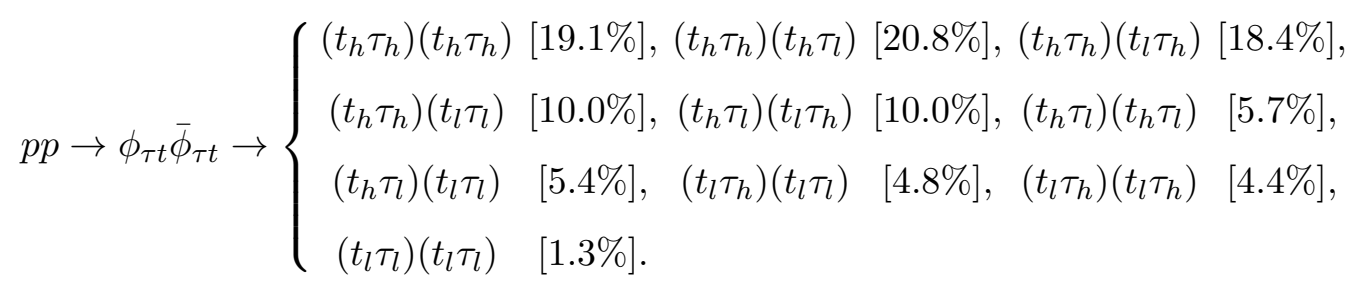

Further complications can arise from $t_{l} \rightarrow \tau \bar{\nu}_{\tau} \bar{b}$ decays where $\tau$ can decay either hadronically or leptonically.

Only one of the many possible final states has been covered. Ref. [60] searched for $p p \rightarrow \phi_{\tau t} \bar{\phi}_{\tau t} \rightarrow\left(t_{h} \tau_{h}^{-}\right)\left(\bar{t}_{l} \tau_{l}^{+}\right)$with $t_{l}$ decays to $e$ or $\mu$ at Run $1\left(8 \mathrm{TeV}, 20 \mathrm{fb}^{-1}\right)$. The mass limit is $m_{S_{\tau t}} \geq 685 \mathrm{GeV}$. Our recast bounds for other MLQs are shown in table 11 . 


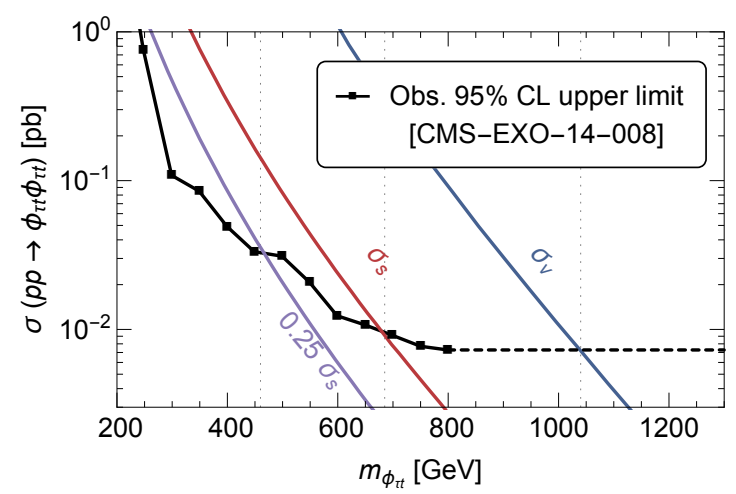

Figure 16. Bounds on scalar and vector MLQs which decay into $(\tau t)(\tau t)$ final states. The observed 95\% CL upper limit on the production cross section times branching fraction (black solid line) is taken from LQ searches in this final state at CMS [60] which stopped at $800 \mathrm{GeV}$. The remainder of the analysis is analogous to figure 8 .

\begin{tabular}{|cc|c|c|}
\hline & & $U^{c} L, U^{c} E^{c}, Q E^{c}$ & $(Q L$ triplet $),(Q L$ singlet $)$ \\
\hline \multirow{2}{*}{$S_{\tau t}$} & $\sigma_{\text {prod }}$ & $\times 1$ & $\times 0.25$ \\
& $m_{S_{\tau t}}$ & $\geq 685 \mathrm{GeV}[60]$ & $\geq 460 \mathrm{GeV}$ \\
\hline
\end{tabular}

\begin{tabular}{|cc|c|}
\hline & & $U^{c \dagger} L, U^{c \dagger} E^{c}, Q^{\dagger} E^{c},\left(Q^{\dagger} L\right.$ triplet $)$ \\
\hline \multirow{2}{*}{$V_{\tau t}$} & $\begin{array}{c}\sigma_{\text {prod }} \\
m_{V_{\tau t}}\end{array}$ & $\times 1$ \\
& $(\geq 1.0 \mathrm{TeV})$ \\
\hline
\end{tabular}

Table 11. 95\% CL lower limits on the mass for various MLQs which decay into $(\tau t)(\tau t)$ final states for scalar LQs (left) and vector LQs (right).

Given that several other final states for $\phi_{\tau t}$ pair-production appear promising, we hope that searches in additional channels will also be performed.

\section{Discussion and summary}

In this paper, we have advocated that LQ pair production searches be organized by the leptoquark matrix. This naturally leads to 9 distinct final states to be searched for. In order to make recasts to arbitrary leptoquark models possible it would be desirable if the experimental collaborations publish bounds on the cross section times branching fraction as a function of LQ mass for each of these final states. We have found strong bounds from existing searches for leptoquarks or equivalent searches for RPV squarks in 6 of the 9 cases. For $(\ell b)(\ell b)$ we have only been able to find a lower mass bound on a particular squark model which only allowed us to obtain recast estimates for other models in this channel. For $(\ell t)(\ell t)$ we were able to obtain bounds by recasting a general purpose cutand-count SUSY search. These bounds could certainly be improved with a more dedicated search. Finally for $(\tau j)(\tau j)$ were not able to find a search which allowed us to obtain reliable strong bounds.

We also note that even in the cases where cross section bounds as a function of mass have been published it would be useful if the search range could be extended as far as possible in LQ mass. This is important because the theoretical cross section times branching fraction for LQs to particular final states can vary by orders of magnitude. At a minimum, the mass range covered should include the range required to set bounds on the MLQ states which can have cross sections times branching fractions ranging from $0.25 \times \sigma_{S}$ to $2 \times \sigma_{V}$. 
We have focused on pair production of LQs with prompt decays. For leptoquarks with large couplings single (and off-shell) production can become important and potentially yield stronger lower bounds on LQ masses. This is especially true when the large coupling is to first generation quarks with their large parton distribution functions. We hope to extend the "Leptoquark Hunter's Guide" to single and off-shell production in a future publication [3]. The LQ matrix is also convenient for organizing the possible single LQ production final states.

Another generalization that we have not covered here is the possibility of displaced vertices from LQ decays with long life times or even LQs that are stable on detector time scales. In the former case exotic new searches using displaced vertices or kinks in tracks would be interesting, in the latter case the LQs would leave strongly ionizing tracks similar to $R$-hadrons in SUSY. For LQs with TeV scale masses, moderate boosts, and decay width $\Gamma=m \lambda^{2} /(16 \pi)$ the vertex displacement is $d \sim 10^{-15} / \lambda^{2} \mathrm{~cm}$. LQs with couplings larger than $\lambda \sim 10^{-6.5}$ have prompt decays with vertex displacements smaller than typical LHC detector impact parameter resolution $\sim 100 \mu \mathrm{m}$. Couplings smaller than $10^{-9}$ lead to "stable" LQs with decays outside the detector.

Is there a theoretically preferred range for the size of couplings? LQs are unlike any particles we have observed so far and therefore we can only make guesses. If they are vectors one should perhaps expect couplings similar to SM gauge couplings, 0.1-1. If they are scalars one might expect couplings similar to SM Yukawa couplings, $10^{-6}-1$. For LQs related to the recent hints for new physics from $B$ meson decays the interesting range of couplings is $10^{-2} \leq \lambda \leq 1$. All these would imply prompt decays but smaller couplings are certainly possible.

Before closing we would like to address a common misconception about leptoquark "generations". It is often said that stringent bounds on flavor violation require the couplings of leptoquarks to be limited to first, second, or third generation leptoquarks. This statement is both incorrect and uses misleading language. What is correct is that large couplings to more than one generation of quarks lead to large quark flavor violation (e.g. $K-\bar{K}$ mixing), and couplings to more than one generation of leptons lead to lepton flavor violation (e.g. $\mu \rightarrow e \gamma$ ). However the generation number of the quark and the lepton do not have to be the same to avoid flavor violation. For example, a leptoquark coupling only to right-handed strange quarks and electrons (in the mass eigenstate basis for the fermions) exactly preserves all relevant U(1) fermion number symmetries (strangeness and electronnumber). Thus flavor constraints do allow the more general form for leptoquark couplings and decays that fill out the full LQ matrix. We discourage the use of the misleading terms "first, second, third generation LQs" and instead suggest the use of more accurate language in which both the quark and lepton generation are specified.

\section{Acknowledgments}

We thank Gudrun Hiller, Simon Knapen, Vojtech Pleskot and Ruth Pöttgen for helpful discussions and comments on the manuscript. BD would like to thank Nicolás Neill, Sebastián Tapia and Alfonso Zerwekh for discussions. MS would like to thank the IAS at 


\begin{tabular}{|cl|}
\hline Symbol & Denotes \\
\hline$S_{\boldsymbol{l} \boldsymbol{q}}$ & Scalar leptoquark, we use the subscript to denote its \\
& leptonic and hadronic decay products. \\
$V_{\boldsymbol{l} \boldsymbol{q}}$ & Vector leptoquark. \\
$\phi_{\boldsymbol{l q}}$ & General leptoquark, either $S$ or $V$. \\
\hline$Q, U^{c}, D^{c}, L, E^{c}$ & SM fermion fields. Subscripts $\{1,2,3\}$ indicate flavor. \\
$N^{c}$ & Dirac partners of the SM left-handed neutrino fields. \\
$\boldsymbol{L}$ & $L, E^{c}, N^{c}$ \\
$\boldsymbol{Q}$ & $Q, U^{c}, D^{c}$ \\
$\hat{Q}, \hat{U}, \hat{D}, \hat{L}, \hat{E}$ & $\mathrm{MSSM}$ fermion superfields which contain the corre- \\
& sponding SM fermion fields. \\
\hline$\nu$ & $\nu_{e}, \nu_{\mu}, \nu_{\tau}$ \\
$j, b$ & light-jet (originating from $u, d, s, c$ or gluons), $b$-jet \\
$\ell$ & $e, \mu$ \\
$\boldsymbol{l}$ & $e, \mu, \tau, \nu$ \\
$\boldsymbol{q}$ & $j, b, t$ \\
\hline
\end{tabular}

Table 12. Summary of notation.

HKUST for its hospitality during the completion of this project. BD was supported partially by Boston University HET visitor program and also partially supported by Conicyt Becas Chile. The work of MS and YZ work is supported by DOE grant DE-SC0015845.

\section{A Notation}

We apologize for our sometimes confusing notation. When referring to the SM fermion fields we use all left-handed 2-component spinors $Q, U^{c}, D^{c}, L, E^{c}, N^{c}$ to reflect the full $\mathrm{SU}(3)_{\text {color }} \times \mathrm{SU}(2)_{\text {weak }} \times \mathrm{U}(1)_{Y}$ symmetry of the SM. The notation uses the fields $Q_{i}$ to denote the (left-handed) quark doublets with $i$ labeling the generation. For example, $Q_{3}=\left(t_{L}, b_{L}\right) . U_{i}^{c}$ and $D_{i}^{c}$ are the charge conjugates of the right-handed quark singlets, again $i$ labeling the generation. For example, $D_{3}^{c}=i \sigma_{2} b_{R}^{*} . L_{i}$ are the lepton doublets and $E_{i}^{c}$ and $N_{i}^{c}$ are three generations of charged lepton and neutrino singlets, respectively. table 12 is a summary of the symbols used in the paper.

We also list the leptoquark nomenclature for the MLQ models used in this paper ( $1^{\text {st }}$ column), their SM gauge quantum numbers $\left(2^{\text {nd }}\right.$ column), the names used in ref. [4] (PDG, $3^{\text {rd }}$ column), and in ref. [2] (BRW, $4^{\text {th }}$ column, see also [66]). Note that in our MLQ models two leptoquarks with the same gauge quantum numbers but different flavor quantum numbers are considered distinct and therefore have different names. In the PDG and BRW notation, LQs with the same gauge quantum numbers are given the same name. That is why the map between our models and PDG and BRW is not one-to-one.

\section{B Comparison between vector and scalar LQ searches}

The kinematics of pair-produced vector LQs is very similar to pair-produced scalar LQs. We generated the processes $p p \rightarrow S_{\mu j} S_{\mu j} \rightarrow\left(j \mu^{+}\right)\left(j \mu^{-}\right)$and $p p \rightarrow V_{\mu j} V_{\mu j} \rightarrow\left(j \mu^{+}\right)\left(j \mu^{-}\right)$ 


\begin{tabular}{|c|c|c|c|c|c|c|c|}
\hline Scalar LQ & $(\mathrm{SU}(3), \mathrm{SU}(2))_{Y}$ & BRW & PDG & Vector LQ & $(\mathrm{SU}(3), \mathrm{SU}(2))_{Y}$ & BRW & PDG \\
\hline$Q L$ triplet & $(3,3)_{-1 / 3}$ & $S_{3}$ & $S_{1}^{\dagger}$ & $Q^{\dagger} L$ triplet & $(\overline{3}, 3)_{-2 / 3}$ & $U_{3}$ & $V_{1}^{\dagger}$ \\
\hline$Q L$ singlet & $(3,1)_{-1 / 3}$ & $S_{1}$ & $S_{0}^{\dagger}$ & $Q^{\dagger} L$ singlet & $(\overline{3}, 1)_{-2 / 3}$ & $U_{1}$ & $V_{0}^{\dagger}$ \\
\hline$U^{c} L$ & $(\overline{3}, 2)_{-7 / 6}$ & $R_{2}$ & $S_{1 / 2}^{\dagger}$ & $U^{c \dagger} L$ & $(3,2)_{1 / 6}$ & $\tilde{V}_{2}$ & $\tilde{V}_{1 / 2}^{\dagger}$ \\
\hline$D^{c} L$ & $(\overline{3}, 2)_{-1 / 6}$ & $\tilde{R}_{2}$ & $\tilde{S}_{1 / 2}^{\dagger}$ & $D^{c \dagger} L$ & $(3,2)_{-5 / 6}$ & $V_{2}$ & $V_{1 / 2}^{\dagger}$ \\
\hline$Q E^{c}$ & $(3,2)_{7 / 6}$ & $R_{2}$ & $S_{1 / 2}^{\dagger}$ & $Q^{\dagger} E^{c}$ & $(\overline{3}, 2)_{5 / 6}$ & $V_{2}$ & $V_{1 / 2}^{\dagger}$ \\
\hline$U^{c} E^{c}$ & $(\overline{3}, 1)_{1 / 3}$ & $S_{1}$ & $S_{0}^{\dagger}$ & $U^{c \dagger} E^{c}$ & $(3,1)_{5 / 3}$ & $\tilde{U}_{1}$ & $\tilde{V}_{0}^{\dagger}$ \\
\hline$D^{c} E^{c}$ & $(\overline{3}, 1)_{4 / 3}$ & $\tilde{S}_{1}$ & $\tilde{S}_{0}^{\dagger}$ & $D^{c \dagger} E^{c}$ & $(3,1)_{2 / 3}$ & $U_{\sim}$ & $V_{0}^{\dagger}$ \\
\hline$Q N^{c}$ & $(3,2)_{1 / 6}$ & $\tilde{R}_{2}$ & - & $Q^{\dagger} N^{c}$ & $(\overline{3}, 2)_{-1 / 6}$ & $\tilde{V}_{2}$ & - \\
\hline$U^{c} N^{c}$ & $(\overline{3}, 1)_{-2 / 3}$ & $\bar{S}_{1}$ & - & $U^{c \dagger} N^{c}$ & $(3,1)_{2 / 3}$ & $U_{1}$ & - \\
\hline$D^{c} N^{c}$ & $(\overline{3}, 1)_{1 / 3}$ & $S_{1}$ & - & $D^{c \dagger} N^{c}$ & $(3,1)_{-1 / 3}$ & $\bar{U}_{1}$ & - \\
\hline
\end{tabular}

Table 13. Alternative LQ names in the literature.

at leading order parton level using MadGraph5 aMC@NLO 2.5.4 which retains polarization effects in production and decays. We then passed the events through the selection cuts for ATLAS' [8] and CMS' [9] analyses. The ratios of the relative efficiencies for various leptoquark masses are shown in figure 2. One sees that the efficiencies for scalar leptoquarks $S$ and vector leptoquarks $V$ are very similar. To better understand the origin of this similarity, we show in figure 17 and figure 18 the scalar and vector distributions for different leptoquark masses, and in the following we detail the variables used:

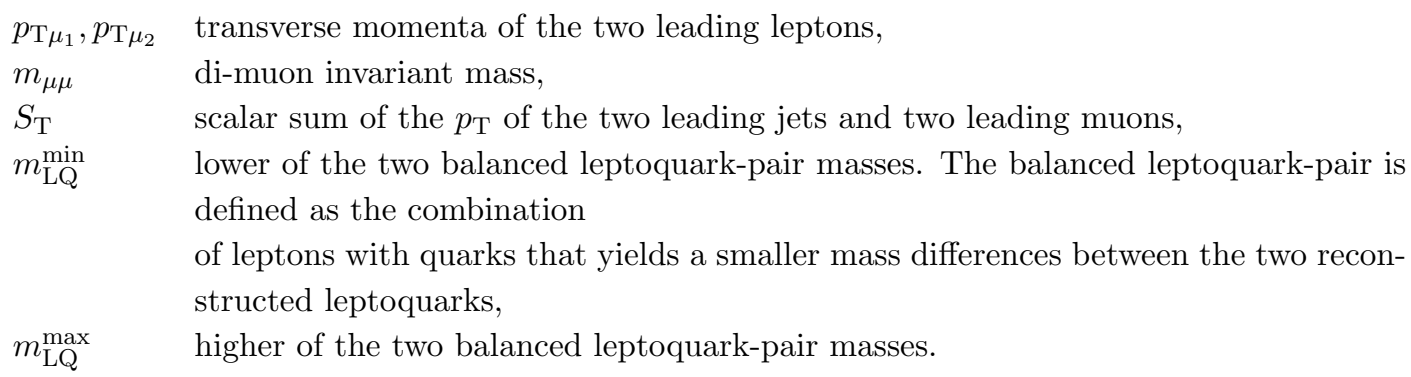

Note that vector LQ production is slightly more forward than scalar LQ production. This results in softer $p_{\mathrm{T}}$ and larger $\eta$ for $V V$ compared to $S S$. Consequently, the relative efficiency for $V V$ decay products to pass through the $p_{\mathrm{T}}$ and $\eta$ cuts are lower than those of $S S$ decay products. This can be seen from the first two panels of figure 2 . The difference is more pronounced for light leptoquarks where also overall efficiencies are smaller, but for $m_{\phi} \geq 500 \mathrm{GeV}$ the difference in efficiencies is always below $10 \%$.

\section{A vector leptoquark model}

Vector leptoquarks may either arise as strongly coupled states within a spectrum of composite resonances from a strongly coupled theory or they may be fundamental, i.e., gauge bosons which obtain masses from spontaneous symmetry breaking. In fact, even composite spin-1 particles must have a spontaneously broken gauge invariance if the compositeness scale is parametrically larger than the mass of the spin-1 particles [67]. In this appendix we 

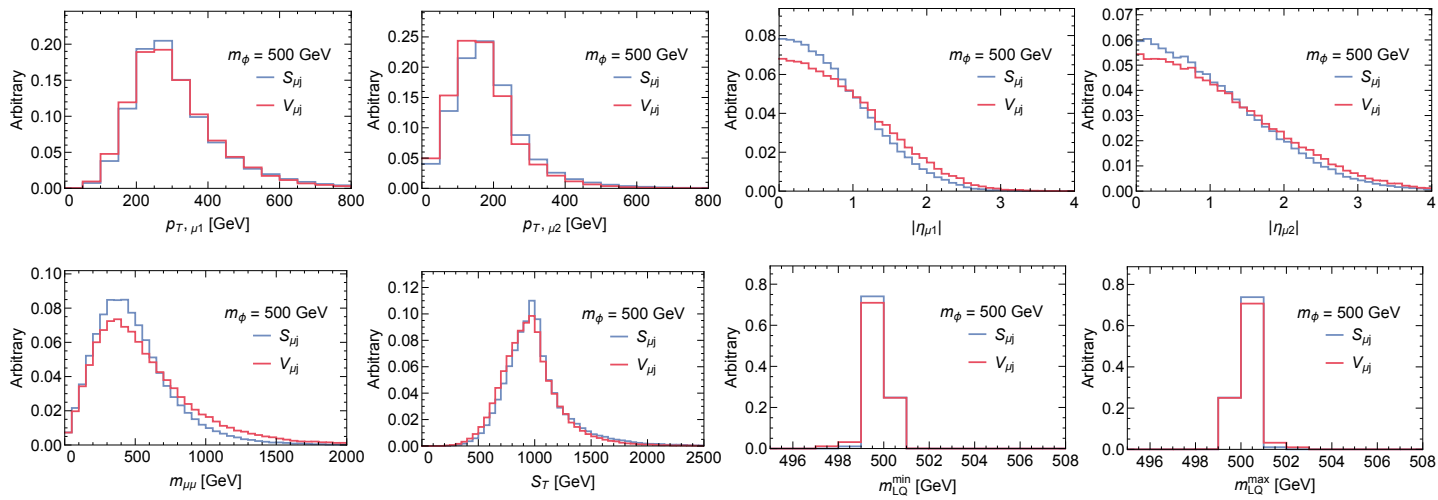

Figure 17. Shape variables for scalar and vector leptoquarks with masses $m_{\phi}=500 \mathrm{GeV}$ after passing the trigger cuts from ATLAS. Reconstructed leptoquark masses are artificially narrow because our parton level analysis does not include detector resolution. Resolution effects would also slightly smoothen the other plots and make vector and scalar distributions even more similar.
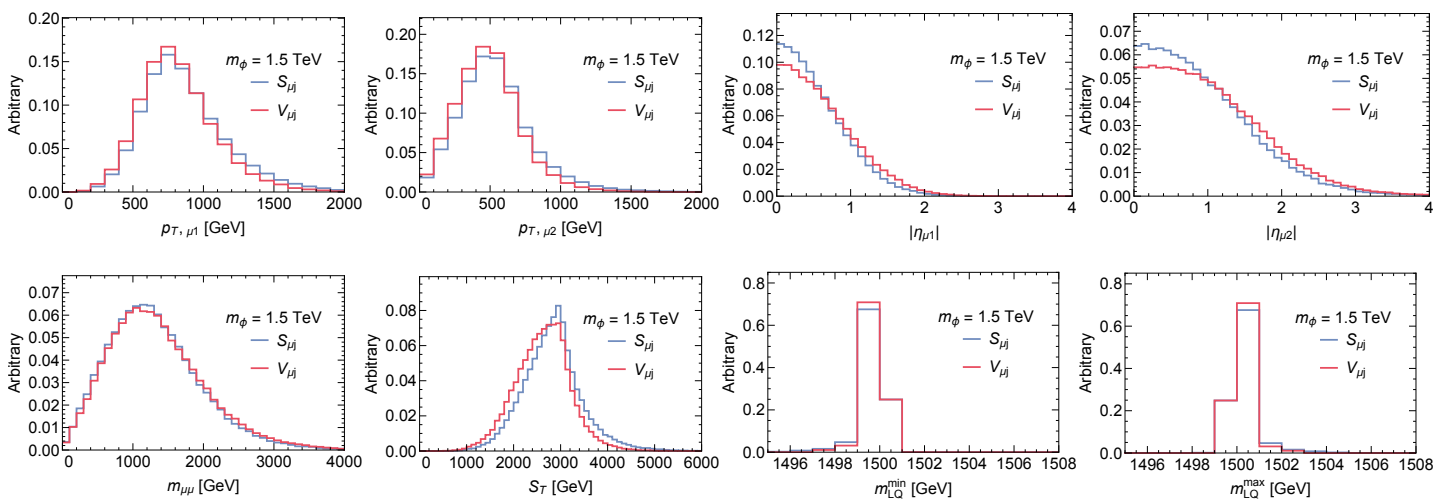

Figure 18. Same as figure 17 for leptoquarks with mass $m_{\phi}=1.5 \mathrm{TeV}$.

\begin{tabular}{|lll|}
\hline & ATLAS & CMS \\
\hline Trigger & At least one muon with $|\eta|<2.5, p_{\mathrm{T}}>26 \mathrm{GeV}$ & At least one muon with \\
& and isolated within $\Delta R=10 \mathrm{GeV} / p_{\mathrm{T}}$ & $p_{\mathrm{T}}>45 \mathrm{GeV},|\eta|<2.1$ \\
& or $p_{\mathrm{T}}>50 \mathrm{GeV}$ without isolation & \\
\hline Muon $p_{\mathrm{T}}, \eta$ & $p_{\mathrm{T} \mu_{1,2}}>40 \mathrm{GeV}$, & $p_{\mathrm{T} \mu_{1,2}}>50 \mathrm{GeV}$, \\
& $\left|\eta_{\mu_{1,2}}\right|<1.01$ or $1.10<\left|\eta_{\mu_{1,2}}\right|<2.5$ & $\left|\eta_{\mu_{1,2}}\right|<2.1$ \\
\hline Jet $p_{\mathrm{T}}, \eta$ & $p_{\mathrm{T} j_{1,2}}>50 \mathrm{GeV}$ & $p_{\mathrm{T} j_{1,2}}>50 \mathrm{GeV}$, \\
& $\left|\eta_{j_{1,2}}\right|<2.8$ & $\left|\eta_{j_{1,2}}\right|<2.4$ \\
\hline Isolation & $\Delta R_{j j, \mu j}>0.4$, & $\Delta R_{j j}>0.4, \Delta R_{\mu j, \mu \mu}>0.3$ \\
& isolated $\mu$ 's with $\Delta R=10 \mathrm{GeV} / p_{\mathrm{T}}$ & \\
\hline$m_{\mu \mu}$ & $>130 \mathrm{GeV}$ & $>50 \mathrm{GeV}$ \\
\hline$S_{\mathrm{T}}$ & $>600 \mathrm{GeV}$ & $>300 \mathrm{GeV}$ \\
\hline
\end{tabular}

Table 14. Summary of selection cuts for ATLAS [8] and CMS [9] searches for pair-produced $S_{\mu j}$. 
present a renormalizable model in which the vector leptoquarks are massive gauge bosons of a spontaneously broken gauge theory.

Leptoquarks carry color and hypercharge, and therefore the gauge generators associated with the leptoquarks must not commute with color and hypercharge. The smallest gauge group which contains color and hypercharge generators and vector leptoquarks is $\mathrm{SU}(4) \times \mathrm{U}(1)_{X} \supset \mathrm{SU}(3) \times \mathrm{U}(1)_{Y}$ where the $\mathrm{U}(1)_{X}$ is necessary to obtain the correct hypercharges for the SM fermions. The spectrum of gauge bosons of this minimal model contains the SM gauge bosons in addition to a complex color triplet leptoquark $V$, and a massive singlet, a $Z^{\prime}$. This $Z^{\prime}$ is a phenomenological problem. The problem is that the $Z^{\prime}$ cannot be much heavier than the leptoquark because their masses have a common origin. The $Z^{\prime}$ couples strongly to SM fermions and therefore has a large production cross section as an $s$-channel resonance. Resonance searches for the $Z^{\prime}$ are much more sensitive probes of this model than leptoquark pair production. In other words, regions of parameter space with potentially discoverable leptoquarks are already ruled out by the non-observation of $Z^{\prime}$ signals at the LHC. This problem with "light" $Z^{\prime}$ resonances is generic to the most simple vector leptoquark models and makes viable models with $\mathrm{TeV}$ scale leptoquark masses non-trivial to construct.

In the following we offer a generic structure for leptoquark models which solves this problem. The trick is familiar from "partial compositeness" models in which heavy gauge bosons arise as composite states from a "strong sector". They obtain small mixing with weakly coupled gauge bosons under which the SM fermions are charged. In this way the heavy "composite" gauge bosons couplings to SM fermions are suppressed by the small mixing angle and direct searches for $Z^{\prime}$ resonances are much less constraining. The UV gauge structure of such models is $\mathrm{SU}(3) \times \mathrm{SU}(2)_{\text {weak }} \times \mathrm{U}(1) \times \mathrm{SU}(N)$ where the $\mathrm{SU}(N)$ is the strongly coupled new gauge group. The SM fermions are all charged under the $\mathrm{SU}(3) \times \mathrm{SU}(2) \times \mathrm{U}(1)$ as usual and carry no $\mathrm{SU}(N)$ charge. The $\mathrm{SU}(N)$ is chosen such that a part of the SM gauge group containing color and hypercharge fits inside $\mathrm{SU}(N)$. The minimal choice is SU(4) but larger groups can also be used. For example, a particularly interesting choice is $\mathrm{SU}(5)$ in which case all the SM gauge bosons (including $\mathrm{SU}(2)_{\text {weak }}$ ) can mix with the heavy gauge bosons. Such a non-minimal structure is also necessary to obtain vector leptoquarks which carry $\mathrm{SU}(2)_{\text {weak }}$ quantum numbers. For definiteness we describe a particular SU(4) model in which the vector leptoquark $V^{\mu}$ couples to the SM fermion bilinear $Q^{\dagger} \bar{\sigma}_{\mu} L$. Here $Q \equiv Q_{i}$ and $L \equiv L_{j}$ can be chosen to be from any generation $i, j=1,2,3$ to give 9 different possible leptoquark couplings. For ease of notation we suppress generation indices in the following. The fields and representations are shown in table 15 .

The gauge group spontaneously breaks at the $\mathrm{TeV}$ scale when a vev for the bifundamental field $\Omega$ develops. It is useful to think of the $\mathrm{SU}(3) \times \mathrm{U}(1)$ gauge groups as a subgroup of a large global SU(4) symmetry. Then the reducible field $\Omega$ transforms as a $\mathbf{4} \otimes \overline{\mathbf{4}}$ representation under this $\mathrm{SU}(4)$ times the gauged $\mathrm{SU}(4)$, and the $\Omega$ vacuum expectation value (vev) breaks the two SU(4) symmetries to the diagonal. This leaves an $\mathrm{SU}(3) \times\left.\mathrm{U}(1)\right|_{\text {diagonal }}=\mathrm{SU}(3)_{\text {color }} \times \mathrm{U}(1)_{Y}$ gauge group unbroken. The vev can be written 


\begin{tabular}{|lcccc|}
\hline Field & $\mathrm{SU}(3)$ & $\mathrm{SU}(2)$ & $\mathrm{U}(1)$ & $\mathrm{SU}(4)$ \\
\hline$Q$ & $\mathbf{3}$ & $\mathbf{2}$ & $1 / 6$ & - \\
$U^{c}$ & $\overline{\mathbf{3}}$ & - & $-2 / 3$ & - \\
$D^{c}$ & $\overline{\mathbf{3}}$ & - & $1 / 3$ & - \\
$L$ & - & $\mathbf{2}$ & $-1 / 2$ & - \\
$E^{c}$ & - & - & 1 & - \\
$H$ & - & $\mathbf{2}$ & $-1 / 2$ & - \\
$\Psi$ & - & $\mathbf{2}$ & - & $\mathbf{4}$ \\
$\Psi^{c}$ & - & $\mathbf{2}$ & - & $\overline{\mathbf{4}}$ \\
$\Omega$ & $(\mathbf{3}, \mathbf{1})$ & - & $(-1 / 6,1 / 2)$ & $\overline{\mathbf{4}}$ \\
\hline
\end{tabular}

Table 15. Fields and representations. There are three generations of each of the SM fermion fields but only one copy of the heavy fermion $\Psi+\bar{\Psi} . H$ is the SM Higgs doublet and $\Omega$ is a "bi-doublet" scalar field which is in the fundamental representation of $\mathrm{SU}(3) \times \mathrm{U}(1)$ (thought of as a subgroup of a larger $\mathrm{SU}(4)$ ) and the anti-fundamental of $\mathrm{SU}(4)$. A TeV scale vev for $\Omega$ is responsible for breaking $[\mathrm{SU}(3) \times \mathrm{U}(1)] \times \mathrm{SU}(4) \rightarrow \mathrm{SU}(3) \times\left.\mathrm{U}(1)\right|_{\text {diagonal }}$.

as a diagonal square matrix

$$
\left[\begin{array}{llll}
f_{3} & & & \\
& & & \\
& f_{3} & & \\
& & f_{3} & \\
& & & f_{1}
\end{array}\right]
$$

The Higgs mechanism generates masses for a full SU(4)'s worth of gauge bosons. The massive gauge bosons are a color octet of massive "gluons" $g^{\prime}$, a $Z^{\prime}$, and the complex color triplet leptoquark $V$. We are interested in the limit of large $g_{4}$, there the vector masses become approximately

$$
\begin{aligned}
& m_{g^{\prime}}=\sqrt{g_{4}^{2}+g_{3}^{2}} f_{3} \stackrel{g_{4} \gg g_{3}}{{ }^{2}} g_{4} f_{3}, \\
& m_{Z^{\prime}}=\frac{1}{2} \sqrt{g_{4}^{2}+2 g_{1}^{2} / 3} \sqrt{f_{3}^{2}+3 f_{1}^{2}} \stackrel{g_{4} \gg g_{1}}{\simeq} \frac{g_{4}}{2} \sqrt{f_{3}^{2}+3 f_{1}^{2}}, \\
& m_{V}=\frac{g_{4}}{\sqrt{2}} \sqrt{f_{3}^{2}+f_{1}^{2}} .
\end{aligned}
$$

These formulas show that by varying $f_{3}$ and $f_{4}$ one can obtain arbitrary leptoquark masses with $m_{g^{\prime}} / m_{V} \in[0, \sqrt{2}]$. The gauge bosons which remain massless after this symmetry breaking are the gluons with gauge coupling $g_{s}=g_{3} g_{4} / \sqrt{g_{4}^{2}+g_{3}^{2}} \simeq g_{3}$ and the hypercharge boson with coupling $g_{Y}=g_{1} g_{4} / \sqrt{g_{4}^{2}+2 g_{1}^{2} / 3} \simeq g_{1}$.

For pair production of the vector leptoquark we need to know the couplings of the leptoquark to gluons. These are given in part by the covariant derivatives in the kinetic term of $V$. But there is another renormalizable coupling between gluons and the leptoquark that is allowed by gauge invariance

$$
\left(1+\kappa_{s}\right) g_{s} G_{\mu \nu}^{a}\left(V^{\mu}\right)^{\dagger} T^{a} V^{\nu},
$$

where $G_{\mu \nu}^{a}$ is the gluon field strength and $T^{a}$ is a Gell-Mann color matrix. The parameter $\kappa_{s}$ is not fixed by gauge invariance of the low energy theory, but it can be derived by 
expanding out the gauge kinetic terms of the unbroken $\mathrm{SU}(3) \times \mathrm{SU}(4)$ gauge theory in terms of the massive and massless vectors. One finds that $\kappa_{s}=0$. The vanishing of $\kappa_{s}$ can also be derived without looking at the full theory by considering unitarity of leptoquark pair production. Only for $\kappa_{s}=0$ does the pair production amplitude $g g \rightarrow V \bar{V}$ have good UV behavior.

In addition, there is a contribution to $q \bar{q} \rightarrow V \bar{V}$ from a diagram with the heavy gluon $g^{\prime}$ as an $s$-channel resonance. The masses of the vectors are such that this resonance is off-shell, but it is required to unitarize the $q \bar{q} \rightarrow V \bar{V}$ pair production amplitude. Therefore we need to determine the couplings of the heavy vectors to fermions. $G^{\prime}$ and $Z^{\prime}$ are the orthogonal linear combinations to the gluons and hypercharge. The $G^{\prime}$ coupling to colored fermions is

$$
\frac{g_{3}^{2}}{\sqrt{g_{3}^{2}+g_{4}^{2}}} \simeq \frac{g_{s}^{2}}{g_{4}} \ll g_{s},
$$

and the $Z^{\prime}$ couplings are proportional to hypercharges times

$$
\frac{g_{1}^{2}}{\sqrt{g_{1}^{2}+3 g_{4}^{2} / 2}} \simeq \sqrt{\frac{2}{3}} \frac{g_{Y}^{2}}{g_{4}} \ll g_{Y} .
$$

We see that the light fermions decouple from the heavy gauge bosons in the limit $g_{4} \gg$ $g_{3}, g_{1}$, making the model safe from precision electroweak constraints and $g^{\prime}$ or $Z^{\prime}$ resonance searches at the LHC.

The $s$-channel diagram for pair production of leptoquarks with intermediate $g^{\prime}$ coupling to leptoquarks is not suppressed in the large $g_{4}$ limit. This is because the coupling of the heavy gluon to the leptoquarks is proportional to $g_{4}$. Then the $s$-channel is proportional to couplings $g_{s}^{2} / g_{4} \times g_{4}=g_{s}^{2}$ just like the QCD diagrams. We included this contribution in figure 4 and varied the mass of $g^{\prime}$ within its allowed limits. In the end, this contribution turns out to not be very important because the large $g g$ parton luminosity enhances the gluon initiated diagrams over the quark initiated ones.

Finally, we consider leptoquark decay through its coupling to light fermions. In the model described so far the coupling vanishes because leptoquark is an $\mathrm{SU}(4)$ gauge boson and the fermions are not charged under SU(4). There is also no mixing of the leptoquark with any of the light gauge bosons. However, it does couple to the heavy fermions $\Psi$. Thus we introduce mixing between $\Psi$ and $Q$ and $L$ of the SM to generate a coupling of $V$ to the light fermions. The relevant mass terms are

$$
\mathcal{L}_{\text {mix }}=\lambda_{\Omega}(Q, L) \Omega \Psi^{c}+M \Psi \Psi^{c}+\text { h.c. }
$$

where - for simplicity - we have imposed a global SU(4) symmetry on the couplings of $Q$ and $L$. In the limit $\lambda_{\Omega}\langle\Omega\rangle \ll M$ the light fermions are still approximately $Q$ and $L$ but with a small $\sin \left(\theta_{\Psi}\right)=\lambda_{\Omega}\langle\Omega\rangle / \sqrt{M^{2}+\left(\lambda_{\Omega}\langle\Omega\rangle\right)^{2}} \simeq \lambda_{\Omega}\langle\Omega\rangle / M$ admixture of $\Psi$. Therefore the effective coupling of the vector leptoquark to light fermions becomes

$$
\lambda=g_{4}\left(\sin \theta_{\Psi}\right)^{2} .
$$

Assuming that $M$ is large enough that the leptoquark does not decay to the heavy fermions this is the only decay mode of the leptoquark, and as long as $\lambda>10^{-7}$ the decay is prompt on detector time scales. 
Open Access. This article is distributed under the terms of the Creative Commons Attribution License (CC-BY 4.0), which permits any use, distribution and reproduction in any medium, provided the original author(s) and source are credited.

\section{References}

[1] B. Schrempp and F. Schrempp, Light leptoquarks, Phys. Lett. B 153 (1985) 101.

[2] W. Buchmüller, R. Ruckl and D. Wyler, Leptoquarks in lepton-quark collisions, Phys. Lett. B 191 (1987) 442 [Erratum ibid. B 448 (1999) 320] [INSPIRE].

[3] S. Foster, M. Schmaltz and Y.-M. Zhong, The leptoquark hunter's guide: single production, to appear.

[4] Particle Data Group collaboration, C. Patrignani et al., Review of particle physics, Chin. Phys. C 40 (2016) 100001 [inSPIRE].

[5] LHCb collaboration, Test of lepton universality using $B^{+} \rightarrow K^{+} \ell^{+} \ell^{-}$decays, Phys. Rev. Lett. 113 (2014) 151601 [arXiv: 1406.6482] [INSPIRE].

[6] LHCb collaboration, Test of lepton universality with $B^{0} \rightarrow K^{* 0} \ell^{+} \ell^{-}$decays, JHEP 08 (2017) 055 [arXiv : 1705.05802] [INSPIRE].

[7] Muon G-2 collaboration, G.W. Bennett et al., Final report of the muon E821 anomalous magnetic moment measurement at BNL, Phys. Rev. D 73 (2006) 072003 [hep-ex/0602035] [INSPIRE].

[8] ATLAS collaboration, Search for scalar leptoquarks in pp collisions at $\sqrt{s}=13 \mathrm{TeV}$ with the ATLAS experiment, New J. Phys. 18 (2016) 093016 [arXiv:1605.06035] [InSPIRE].

[9] CMS collaboration, Search for pair-production of second-generation scalar leptoquarks in pp collisions at $\sqrt{s}=13 \mathrm{TeV}$ with the CMS detector, CMS-PAS-EXO-16-007 (2016).

[10] I. Dorsner, S. Fajfer and A. Greljo, Cornering scalar leptoquarks at LHC, JHEP 10 (2014) 154 [arXiv: 1406.4831] [INSPIRE].

[11] N. Raj, Anticipating nonresonant new physics in dilepton angular spectra at the LHC, Phys. Rev. D 95 (2017) 015011 [arXiv: 1610.03795] [INSPIRE].

[12] M. Krämer, T. Plehn, M. Spira and P.M. Zerwas, Pair production of scalar leptoquarks at the Tevatron, Phys. Rev. Lett. 79 (1997) 341 [hep-ph/9704322] [INSPIRE].

[13] M. Krämer, T. Plehn, M. Spira and P.M. Zerwas, Pair production of scalar leptoquarks at the CERN LHC, Phys. Rev. D 71 (2005) 057503 [hep-ph/0411038] [INSPIRE].

[14] J. Blumlein, E. Boos and A. Kryukov, Leptoquark pair production in hadronic interactions, Z. Phys. C 76 (1997) 137 [hep-ph/9610408] [INSPIRE].

[15] J. Blumlein, E. Boos and A. Kryukov, Leptoquark pair production cross-sections at hadron colliders, hep-ph/9811271 [inSPIRE].

[16] T. Mandal, S. Mitra and S. Seth, Pair production of scalar leptoquarks at the LHC to NLO parton shower accuracy, Phys. Rev. D 93 (2016) 035018 [arXiv:1506.07369] [INSPIRE].

[17] ATLAS collaboration, A search for B-L R-parity-violating scalar tops in $\sqrt{s}=13 \mathrm{TeV} p p$ collisions with the ATLAS experiment, ATLAS-CONF-2017-036 (2017).

[18] CMS collaboration, Search for supersymmetry in hadronic final states with missing transverse energy using the variables $\alpha_{T}$ and b-quark multiplicity in pp collisions at $\sqrt{s}=8$ TeV, Eur. Phys. J. C 73 (2013) 2568 [arXiv: 1303.2985] [InSPIRE]. 
[19] CMS collaboration, Searches for supersymmetry using the $M_{T 2}$ variable in hadronic events produced in pp collisions at $8 \mathrm{TeV}$, JHEP 05 (2015) 078 [arXiv: 1502.04358] [INSPIRE].

[20] CMS collaboration, Search for supersymmetry in multijet events with missing transverse momentum in proton-proton collisions at $13 \mathrm{TeV}$, CMS-PAS-SUS-16-033 (CMS Collaboration).

[21] CMS collaboration, Search for new physics in the all-hadronic final state with the MT2 variable, CMS-PAS-SUS-16-036 (2016).

[22] CMS collaboration, Search for supersymmetry in multijet events with missing transverse momentum in proton-proton collisions at 13 TeV, Phys. Rev. D 96 (2017) 032003 [arXiv: 1704.07781] [INSPIRE].

[23] ATLAS collaboration, Search for scalar charm quark pair production in pp collisions at $\sqrt{s}=8 \mathrm{TeV}$ with the ATLAS detector, Phys. Rev. Lett. 114 (2015) 161801 [arXiv: 1501.01325] [INSPIRE].

[24] ATLAS collaboration, Search for squarks and gluinos in final states with jets and missing transverse momentum using $36 \mathrm{fb}^{-1}$ of $\sqrt{\mathrm{s}}=13 \mathrm{TeV}$ pp collision data with the ATLAS detector, ATLAS-CONF-2017-022 (2017).

[25] ATLAS collaboration, Search for direct third-generation squark pair production in final states with missing transverse momentum and two b-jets in $\sqrt{s}=8 \mathrm{TeV}$ pp collisions with the ATLAS detector, JHEP 10 (2013) 189 [arXiv:1308.2631] [INSPIRE].

[26] ATLAS collaboration, Searches for scalar leptoquarks in pp collisions at $\sqrt{s}=8 \mathrm{TeV}$ with the ATLAS detector, Eur. Phys. J. C 76 (2016) 5 [arXiv:1508.04735] [InSPIRE].

[27] ATLAS collaboration, ATLAS Run 1 searches for direct pair production of third-generation squarks at the Large Hadron Collider, Eur. Phys. J. C 75 (2015) 510 [arXiv:1506.08616] [INSPIRE].

[28] ATLAS collaboration, Search for bottom squark pair production in proton-proton collisions at $\sqrt{s}=13 \mathrm{TeV}$ with the ATLAS detector, Eur. Phys. J. C 76 (2016) 547 [arXiv: 1606.08772] [INSPIRE].

[29] CMS collaboration, Searches for pair production of third-generation squarks in $\sqrt{s}=13 \mathrm{TeV}$ pp collisions, Eur. Phys. J. C 77 (2017) 327 [arXiv: 1612.03877] [INSPIRE].

[30] ATLAS collaboration, Search for supersymmetry in events with b-tagged jets and missing transverse energy in pp collisions at $\sqrt{s}=13 \mathrm{TeV}$ with the ATLAS detector, ATLAS-CONF-2017-038 (2017).

[31] CMS collaboration, Search for top-squark pair production in the single-lepton final state in pp collisions at $\sqrt{s}=8 \mathrm{TeV}$, Eur. Phys. J. C 73 (2013) 2677 [arXiv:1308.1586] [InSPIRE].

[32] ATLAS collaboration, Search for top squark pair production in final states with one isolated lepton, jets and missing transverse momentum in $\sqrt{s}=8 \mathrm{TeV} p p$ collisions with the ATLAS detector, JHEP 11 (2014) 118 [arXiv:1407.0583] [INSPIRE].

[33] ATLAS collaboration, Search for the supersymmetric partner of the top quark in the jets+Emiss final state at $\sqrt{s}=13 \mathrm{TeV}$, ATLAS-CONF-2016-077 (2016).

[34] CMS collaboration, Search for top squark pair production in compressed-mass-spectrum scenarios in proton-proton collisions at $\sqrt{s}=8 \mathrm{TeV}$ using the $\alpha_{T}$ variable, Phys. Lett. B 767 (2017) 403 [arXiv: 1605. 08993] [INSPIRE]. 
[35] CMS collaboration, Search for direct pair production of supersymmetric top quarks decaying to all-hadronic final states in pp collisions at $\sqrt{s}=8 \mathrm{TeV}$, Eur. Phys. J. C76 (2016) 460, [arXiv: 1603.00765].

[36] CMS collaboration, Search for direct pair production of scalar top quarks in the single- and dilepton channels in proton-proton collisions at $\sqrt{s}=8 \mathrm{TeV}$, JHEP 07 (2016) 027 [Erratum ibid. 09 (2016) 056] [arXiv: 1602.03169] [INSPIRE].

[37] ATLAS collaboration, Search for a scalar partner of the top quark in the jets + ETmiss final state at $\sqrt{s}=13 \mathrm{TeV}$ with the ATLAS detector, ATLAS-CONF-2017-020 (2017).

[38] ATLAS collaboration, Search for new phenomena in events with missing transverse momentum and a Higgs boson decaying into two photons at $\sqrt{s}=13$ TeV with the ATLAS detector, ATLAS-CONF-2017-024 (2017).

[39] CMS collaboration, Search for direct stop pair production in the dilepton final state at $\sqrt{s}=13 \mathrm{TeV}$, CMS-PAS-SUS-17-001 (2017).

[40] CMS Collaboration, Search for direct top squark pair production in the all-hadronic final state in proton-proton collisions at $\sqrt{s}=13 \mathrm{TeV}$, CMS-PAS-SUS-16-049 (2016).

[41] CMS collaboration, Search for direct production of supersymmetric partners of the top quark in the all-jets final state in proton-proton collisions at $\sqrt{s}=13 \mathrm{TeV}$, JHEP 10 (2017) 005 [arXiv: 1707.03316] [INSPIRE].

[42] CMS collaboration, Search for pair production of second-generation scalar leptoquarks in pp collisions at $\sqrt{s}=7 \mathrm{TeV}$, Phys. Rev. Lett. 106 (2011) 201803 [arXiv:1012.4033] [INSPIRE].

[43] CMS collaboration, Search for pair production of first-generation scalar leptoquarks in pp collisions at $\sqrt{s}=7 \mathrm{TeV}$, Phys. Rev. Lett. 106 (2011) 201802 [arXiv:1012.4031] [INSPIRE].

[44] ATLAS collaboration, Search for first generation scalar leptoquarks in pp collisions at $\sqrt{s}=7 \mathrm{TeV}$ with the ATLAS detector, Phys. Lett. B 709 (2012) 158 [Erratum ibid. B 711 (2012) 442] [arXiv: 1112.4828] [INSPIRE].

[45] ATLAS collaboration, Search for pair production of first or second generation leptoquarks in proton-proton collisions at $\sqrt{s}=7 \mathrm{TeV}$ using the ATLAS detector at the LHC, Phys. Rev. D 83 (2011) 112006 [arXiv: 1104.4481] [INSPIRE].

[46] CMS collaboration, Search for first generation scalar leptoquarks in the evjj channel in pp collisions at $\sqrt{s}=7 \mathrm{TeV}$, Phys. Lett. B 703 (2011) 246 [arXiv:1105.5237] [InSPIRE].

[47] ATLAS collaboration, Search for second generation scalar leptoquarks in pp collisions at $\sqrt{s}=7 \mathrm{TeV}$ with the ATLAS detector, Eur. Phys. J. C 72 (2012) 2151 [arXiv:1203.3172] [INSPIRE].

[48] CMS collaboration, Search for pair production of first- and second-generation scalar leptoquarks in pp collisions at $\sqrt{s}=7$ TeV, Phys. Rev. D 86 (2012) 052013 [arXiv: 1207.5406] [INSPIRE].

[49] CMS collaboration, Search for pair-production of first generation scalar leptoquarks in $p p$ collisions at $\sqrt{s}=8 \mathrm{TeV}$, CMS-PAS-EXO-12-041 (2012).

[50] CMS collaboration, Search for pair-production of second generation leptoquarks in $8 \mathrm{TeV}$ proton-proton collisions, CMS-PAS-EXO-12-042 (2012).

[51] CMS collaboration, Search for pair production of first and second generation leptoquarks in proton-proton collisions at $\sqrt{s}=8 \mathrm{TeV}$, Phys. Rev. D 93 (2016) 032004 [arXiv:1509.03744] [INSPIRE]. 
[52] CMS collaboration, Search for pair-production of first generation scalar leptoquarks in $p p$ collisions at $\sqrt{s}=13$ TeV with $2.6 \mathrm{fb}^{-1}$, CMS-PAS-EXO-16-043 (2016).

[53] ATLAS collaboration, $A$ search for $B-L R$-parity violating scalar top decays in $\sqrt{s}=8$ TeV pp collisions with the ATLAS experiment, ATLAS-CONF-2015-015 (2015).

[54] CMS collaboration, Search for pair production of third-generation leptoquarks and top squarks in pp collisions at $\sqrt{s}=7$ TeV, Phys. Rev. Lett. 110 (2013) 081801 [arXiv: 1210.5629] [INSPIRE].

[55] CMS collaboration, Search for third-generation leptoquarks and scalar bottom quarks in pp collisions at $\sqrt{s}=7 \mathrm{TeV}$, JHEP 12 (2012) 055 [arXiv:1210.5627] [INSPIRE].

[56] ATLAS collaboration, Search for third generation scalar leptoquarks in pp collisions at $\sqrt{s}=7$ TeV with the ATLAS detector, JHEP 06 (2013) 033 [arXiv: 1303.0526] [INSPIRE].

[57] CMS collaboration, Search for pair production of third-generation scalar leptoquarks and top squarks in proton-proton collisions at $\sqrt{s}=8 \mathrm{TeV}$, Phys. Lett. B 739 (2014) 229 [arXiv: 1408.0806] [INSPIRE].

[58] CMS collaboration, Search for heavy neutrinos or third-generation leptoquarks in final states with two hadronically decaying $\tau$ leptons and two jets in proton-proton collisions at $\sqrt{s}=13 \mathrm{TeV}$, JHEP 03 (2017) 077 [arXiv: 1612.01190] [INSPIRE].

[59] CMS collaboration, Search for third-generation scalar leptoquarks and heavy right-handed neutrinos in final states with two tau leptons and two jets in proton-proton collisions at $\sqrt{s}=13 \mathrm{TeV}$, JHEP 07 (2017) 121 [arXiv: 1703.03995] [INSPIRE].

[60] CMS collaboration, Search for third-generation scalar leptoquarks in the t $\tau$ channel in proton-proton collisions at $\sqrt{s}=8 \mathrm{TeV}$, JHEP 07 (2015) 042 [Erratum ibid. 11 (2016) 056] [arXiv: 1503.09049] [INSPIRE].

[61] B. Gripaios, A. Papaefstathiou, K. Sakurai and B. Webber, Searching for third-generation composite leptoquarks at the LHC, JHEP 01 (2011) 156 [arXiv:1010.3962] [INSPIRE].

[62] CMS collaboration, Identification of c-quark jets at the CMS experiment, CMS-PAS-BTV-16-001 (2016).

[63] CMS collaboration, Search for single production of scalar leptoquarks in proton-proton collisions at $\sqrt{s}=8 \mathrm{TeV}$, Phys. Rev. D 93 (2016) 032005 [arXiv:1509.03750] [InSPIRE].

[64] CMS collaboration, Search for new physics with multileptons and jets in $35.9 \mathrm{fb}^{-1}$ of $\mathrm{pp}$ collision data at $\sqrt{s}=13$ TeV, CMS-PAS-SUS-16-041 (2016).

[65] J. Alwall, R. Frederix, S. Frixione, V. Hirschi, F. Maltoni, O. Mattelaer et al., The automated computation of tree-level and next-to-leading order differential cross sections and their matching to parton shower simulations, JHEP 07 (2014) 079 [arXiv: 1405.0301] [INSPIRE].

[66] I. Doršner, S. Fajfer, A. Greljo, J.F. Kamenik and N. Košnik, Physics of leptoquarks in precision experiments and at particle colliders, Phys. Rept. 641 (2016) 1 [arXiv:1603.04993] [INSPIRE].

[67] S. Weinberg and E. Witten, Limits on massless particles, Phys. Lett. 96B (1980) 59 [INSPIRE]. 